\title{
Power Control Optimization for Large-Scale Multi-Antenna Systems
}

\author{
Haijun $\mathrm{Yu}^{\dagger}$, Zhenyu Zhou ${ }^{\dagger}$, Shahid Mumtaz ${ }^{\ddagger}$, Saba Al-Rubaye*, Antonios Tsourdos*, \\ and Rose Qingyang $\mathrm{Hu}^{\S}$ \\ ${ }^{\dagger}$ North China Electric Power University, China \\ ${ }^{\ddagger}$ Instituto de Telecomunicações, Portugal \\ ${ }^{*}$ School of Aerospace, Transport and Manufacturing, Cranfield University, UK \\ ${ }^{\S}$ Electrical and Computer Engineering, Utah State University, USA
}

\begin{abstract}
Large-scale multi-antenna systems can effectively improve data transmission reliability and throughput for smart grid. However, the massive number of antennas and radio frequency (RF) chains also result in high complexity and energy cost. In this paper, we develop a new performance benchmark named energy economic efficiency for measuring the time-average throughput per energy cost. Then, we investigate how to maximize long-term energy economic efficiency via the joint optimization of communication and energy resource allocation. The formulated joint optimization problem is NP-hard because it not only involves long-term nonlinear optimization objective and constraints, but also involves both integer and continuous optimization variables. Next, we propose an online joint antenna selection and power control algorithm by combining nonlinear fractional programming, Lyapunov optimization, and bisection method. The proposed algorithm can achieve bounded performance deviation from the optimum performance without requiring the prior knowledge of future channel state information (CSI), energy arrival, and electricity price. Finally, a comprehensive theoretical analysis is provided, and the proposed algorithm is verified through simulations under various system configurations.
\end{abstract}

\section{Index Terms}

Large-scale multi-antenna systems, Lyapunov optimization, smart grid, energy economic efficiency, bisection method, nonlinear fractional programming.

\section{INTRODUCTION}

Smart grid relies on advanced communication technologies for improving efficiency, reliability, and economics of traditional power grid. To achieve intelligent management of energy generation, 
transmission, transformation, delivery, and storage, huge volume of data have to be transmitted on a real-time basis. With the development of $5 \mathrm{G}$ cellular technologies, large-scale multi-antenna systems with hundreds of antennas have appeared as a promising solution. It can effectively improve data transmission reliability and throughput for smart grid via the exploitation of antenna gain [1]-[3]. The basic principles of large-scale multi-antenna systems were investigated [4], and real-world testbeds were developed [5], [6]. Despite its huge benefits, the massive number of antennas and RF chains also raises new challenges such as high complexity and power consumption. For each antenna, a separate radio frequency (RF) chain is required for signal processing, which is generally more expensive and energy consuming than the antenna itself [7], [8]. The overall energy costs can no longer be neglected as in existing cellular systems.

The costs of energy consumption can be reduced from both the energy and communication domains [9]. In the energy domain, a possible solution is to exploit external renewable energy sources such as wind and solar energy. Smart grid enables seamless integration of renewable energy sources into the demand side, thereby reducing the amount of grid power consumption. In the communication domain, the active number of antennas and respective RF chains can also be reduced through antenna selection. Previous experiment results have demonstrated that the contributions of different antennas are not equal in real-world communication channels [5]. Therefore, instead of using all antennas, it is beneficial to only select those antennas which contribute the most while turning off the rest.

However, the research on large-scale multi-antenna systems for smart grid is still in its infancy. There exist several key challenges that remain unsolved. First of all, energy resource allocation and communication resource allocation are intertwined with each other and should be jointly optimized. The formulated joint resource allocation problem is NP-hard due to the coupling between energy and communication domains. Second, renewable energy sources with intermittent and fluctuating characteristics and capacity-constrained energy storage devices cannot provide reliable quality of service (QoS) guarantees. A more feasible approach is to utilize both unreliable renewable energy sources and reliable grid power in a complementary manner [10]. The coexistence of various energy sources further complicates the resource allocation problem. Last but not least, the long-term system performance depends on the precise knowledge of future electricity price, energy arrival and channel state information (CSI), which is generally unavailable. In real-world implementation, even the accurate statistical information is difficult to be identified. 
To address these challenges, we propose an online joint resource allocation algorithm by combining nonlinear fractional programming, bisection method, and Lyapunov optimization. Its main goal is to maximize the long-term energy economic efficiency via the joint optimization of communication resource allocation, i.e., antenna selection, and energy resource allocation, i.e., power control. First, we formulate the energy economic efficiency maximization problem as a joint antenna selection and power control problem over an infinite horizon, which cannot be solved in polynomial time. Both long-term and short-term constraints are taken into account. Second, the formulated problem in fractional form is transformed into an equivalent subtractiveform problem based on nonlinear fractional programming. Then, the long-term stochastic optimization problem is further converted to a short-term deterministic optimization problem by leveraging Lyapunov optimization. Third, by opportunistically minimizing the upper bound of drift-minus-reward, the antenna selection and power control subproblems are separated and solved sequentially. The main contributions of this work are summarized as follows:

- Energy economic efficiency maximization: We consider a new performance metric named energy economic efficiency, which provides a benchmark for measuring the time-average throughput per unit energy cost. Compared to other metrics such as energy efficiency, it is more suitable for the smart grid environment with various energy sources and electricity prices. Particularly, energy efficiency can be abstracted as a special case of this new metric.

- Long-term online optimization with bounded performance deviation: The proposed algorithm is aware of long-term optimization objective and constraints. It can guarantee bounded deviation from the optimum performance without requiring any prior knowledge of future CSI, energy arrival, and electricity prices.

- Comprehensive theoretical analysis and performance validation: We provide a comprehensive theoretical analysis for the proposed algorithm in terms of reliability, optimality, and complexity. Intensive simulation results are conducted under different scenarios to demonstrate its performance gains.

The rest of the paper is outlined as follows. A survey of related works is presented in Section II. Section III introduces the system model in details. The problem formulation is provided in Section IV. Section V elaborates the proposed online resource allocation algorithm. A comprehensive property analysis is provided in Section VI. Numerical results and analysis are introduced in Section VII. Finally, the conclusion is summarized in Section VIII. 


\section{RELATED WORK}

When implementing large-scale multi-antenna system for the smart grid, both the communication resource allocation and energy resource allocation should be jointly optimized according to dynamic CSI, energy arrival, and electricity prices. The key research challenge is that these uncertain factors may cause a high level of volatility and increase potential performance disturbances.

For the optimization of communication resources, antenna selection techniques for conventional multi-antenna systems are summarized in [7], and were then extended to co-located largescale multi-antenna systems [5], [11], [12], as well as distributed antenna systems (DAS) [13], [14]. In [11], Li et al. proposed a bisection-based antenna selection algorithm to maximize the energy efficiency for the large-scale multi-antenna systems. In [5], Gao et al. evaluated antenna selection performance for large-scale multi-antenna systems with 128 elements and proposed a close-to-optimal antenna selection algorithm based on measurements of the received power. Amadori et al. developed an antenna selection algorithm to maximize the received power by identifying antennas with higher constructive interference [12]. Nevertheless, these attempts are only valid for scenarios with constant energy supply, and have not considered the smart grid scenarios with dynamic energy arrival and electricity prices.

For the optimization of energy resources, power control in renewable energy based communication systems was originally developed for nonfading channels [15], and was then extended to broadcasting channels [16], multiple-hop relay channels [17], [18], fading channels [19], and multiple input multiple output (MIMO) channels [20]. However, most of these attempts only target the power control problem in systems with a limited number of antennas. The specific characteristics of large-scale multi-antenna systems are largely neglected, and antenna selection is not jointly optimized with power control.

Markov decision process (MDP) provides an effective approach to handle uncertainties, in which the uncertain parameters are assumed to follow a well-known probability distribution [21][24]. In [21], Stephen et al. modeled the joint optimization problem of pilot allocation and antenna selection as a partially observed MDP, and developed a joint resource allocation algorithm to maximize the expected throughput. The drawback is that the performance degrades dramatically if the practical probability distributions of uncertain factors are different from the presumed statistical models. In addition, the computation complexity of MDP is enormously high because 
of the well-known "curse of dimensionality" [25]. Although the algorithmic structures of lowcomplexity sub-optimal solutions were developed [15]-[17], [20], the knowledge of statistical information is required as a priori.

An alternative low-complexity sub-optimal solution for addressing long-term stochastic optimization problems is Lyapunov optimization [26]. It allows a distribution-free model of uncertain factors and provides bounded performance guarantees under all possible realizations of uncertainties. Some researchers have already applied it for resource allocation optimization in large-scale multi-antenna systems. In [27], Jiang et al. studied the joint user scheduling and beam selection problem in large-scale multi-antenna systems, and proposed an online sum-rate maximization algorithm based on Lyapunov optimization. The same authors extended their work to the scenario where statistical CSI is available [26]. Nevertheless, these works are not suitable for the smart grid environment with dynamic energy arrival and electricity prices. In [28], Mao et al. considered the intermittent characteristics of renewable energy sources and proposed a Lyapunov optimization-based base station (BS) assignment and power control (BAPC) algorithm to minimize the network service cost. Nevertheless, it is not suitable for large-scale multi-antenna systems considered in this paper.

Renewable energy sources based large-scale multi-antenna systems have attracted intensive studies from both academia and industry [29]-[34]. In [29], Demir et al. proposed a joint antenna and hybrid beamforming algorithm for multi-user relay systems based on simultaneous wireless information and power transfer (SWIPT). The relationship between SWIPT-based power transfer and throughput was studied in [30]. In [34], Men et al. proposed a joint relay and antenna selection algorithm based on the power splitting-based relaying (PSR) protocol to minimize the system outage probability. However, the SWIPT-based large-scale multi-antenna systems are quite different from our work since the main focus is on how to effectively transfer energy from the BS to terminals. In [31], Lei et al. studied the user association and power control problem in renewable energy-based heterogeneous networks, and proposed a low-complexity precoding scheme for co-channel interference cancellation. A geometric water-filling resource allocation algorithm with group upper bounded power constraints and recursion machinery was proposed to maximize the system throughput in [32]. However, most of these previous works assume that energy harvesting follows some well-known statistical models, and require the precise statistical information of energy arrival. In our previous work [33], we proposed an offline joint antenna selection and power allocation algorithm to maximize the energy efficiency of renewable energy 

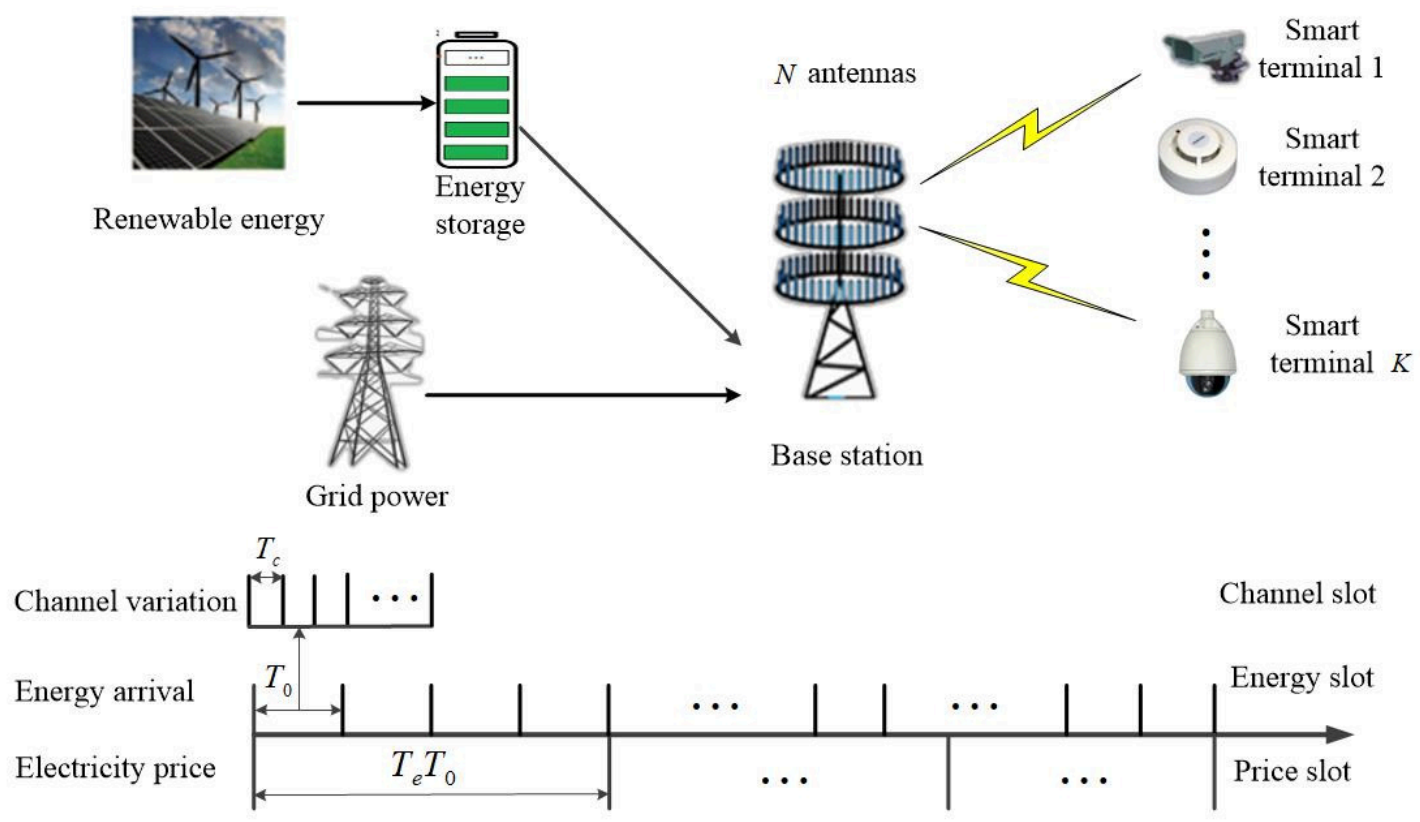

Fig. 1. System model

sources based large-scale multi-antenna systems. It requires prefect knowledge of future energy arrival and CSI, which is infeasible for practical implementation.

Different from above research attempts, we investigate how to jointly optimize antenna selection and power control in large-scale multi-antenna systems according to dynamic energy arrival, CSI, and electricity prices, which has not been considered before. Compared with [29][34], we consider a more practical scenario where even the statistical knowledge of uncertainties is unknown. Furthermore, we emphasize on a new performance metric named energy economic efficiency, which provides an effective benchmark for measuring the time-average throughput per unit energy cost. This new objective involves long-term optimization of a nonlinear fractional programming problem with both integer and continuous variables, which is not considered in [29]-[34]. 


\section{System MODEL}

We consider a typical downlink large-scale multi-antenna systems for smart grid, which is illustrated in Fig. 1. There are one BS and $K$ smart terminals (STs). The BS is powered by two complementary energy sources including the fluctuating renewable energy sources and the reliable power grid. The renewable energy harvested from the external environment is stored in a capacity-constrained battery before usage, while the energy supply variability is compensated by the grid power. In the following, the models of energy arrival, electricity price, data transmission, and power consumption are introduced in details.

\section{A. Dynamic Energy Arrival and Electricity Price Models}

The dynamic process of renewable energy arrival and real-time electricity price over the continuous timeline is illustrated in Fig. 1. The time slot model proposed in [35], [36] is adopted. Specifically, we partition the time dimension into successive identical energy slots with slot duration $T_{0}$ seconds, which are indexed by $t_{e}=1,2, \cdots \infty$. The time-scale difference among energy arrival, CSI variation, and electricity price variation has also been taken into consideration. Generally, it is assumed that CSI varies much faster than that of energy arrival, while electricity price varies much slower than that of energy arrival, i.e., electricity prices can be assumed as a deterministic value during a large number of energy slots. Therefore, each energy slot consists of $T_{0} / T_{c}$ channel slots, where $T_{c}$ is channel slot duration, and multiple energy slots, e.g., $T_{e}$ slots, are grouped as a price slot with duration of $T_{e} T_{0}$ seconds, which is indexed by $t_{w}=1,2, \cdots, \infty$. It is noted that in real-world implementations, $\frac{T_{0}}{T_{c}}$ may not be an integer. The proposed algorithm can still be applicable since antenna selection and power control are jointly optimized at each energy slot instead of each channel slot.

In the smart grid, both energy arrival and electricity prices are varying temporally and spatially. Let $E_{i n}\left(t_{e}\right) \in\left[0, E_{\max }\right]$ denote the amount of energy arrives to the battery at the $t_{e}$-th slot, which is i.i.d. over energy slots. Let $\omega^{E}\left(t_{\omega}\right) \in\left[0, \omega_{\text {max }}^{E}\right]$ and $\omega^{G}\left(t_{\omega}\right) \in\left[0, \omega_{\text {max }}^{G}\right]$ denote the prices of renewable energy and grid power at the $t_{\omega}$-th slot, which are i.i.d. over price slots, respectively. We can set $0<\omega^{E}<\omega^{G}$ to encourage the consumption of renewable energy.

Let $B\left(t_{e}\right)$ denote the battery state and let $P_{\text {total }}^{E}\left(t_{e}\right)$ denote the renewable power consumption at the $t_{e}$-th slot, respectively. Since the energy consumed at each slot cannot exceed the energy 
stored in the battery, we have the energy causality constraint:

$$
P_{\text {total }}^{E}\left(t_{e}\right) T_{0} \leq B\left(t_{e}\right)
$$

Then, the battery state can be modeled as a dynamic energy queue, where energy arrival and energy consumption represent the input and output of the queue, respectively. The energy queue is updated as

$$
B\left(t_{e}+1\right)=\min \left\{B\left(t_{e}\right)-P_{\text {total }}^{E}\left(t_{e}\right) T_{0}+E_{\text {in }}\left(t_{e}\right), B_{\text {max }}\right\}
$$

where $B_{\max }$ denotes the battery capacity.

Remark 1: Compared with previous works [33], [35], [37], we have not put any restriction on the statistical distributions of uncertain factors such as energy arrival and electricity price. In other words, the proposed joint resource allocation algorithm can be applicable even when the knowledge of statistical distributions is unknown.

\section{B. Data Transmission Model}

The BS is assumed to be equipped with a total of $N$ antennas $(N>>1)$, while each $\mathrm{ST}$ is based on a single antenna due to space and cost limitations. The downlink signal received at the terminal side is written as

$$
y=\mathbf{H}^{H} \mathbf{W} \mathbf{x}+n_{0},
$$

where $\mathbf{x}$ is the $K \times 1$ transmission symbol vector, and $\mathbf{W}$ is the $N \times K$ precoding matrix. In this paper, we adopt the zero forcing (ZF) precoding scheme, i.e., $\mathbf{W}=\mathbf{H}\left(\mathbf{H}^{H} \mathbf{H}\right)^{-1}$, due to its superior performance and low complexity when $N>>K[38] . \mathbf{H}=\left[\mathbf{h}_{1}, \mathbf{h}_{2}, \ldots, \mathbf{h}_{K}\right]$ is the $N \times K$ matrix of channel gain, and each element $\mathbf{h}_{k}=\left[h_{k, 1}, h_{k, 2}, \ldots, h_{k, N}\right]^{T}$ denotes the $N$ dimensional vector of channel gain between the BS and the $k$-th ST. $n_{0}$ is additive white Gaussian noise (AWGN) which follows a normal distribution $\mathcal{N}\left(0, \sigma^{2} \mathbf{I}_{\mathbf{k}}\right)$, where $\mathbf{I}_{\mathbf{k}}$ is the $N \times K$ identity matrix. Following the channel model assumptions used in [11], [39]-[41], we mainly consider resource allocation optimization based on the small-scale channel fading, and the elements in $\mathbf{H}$ are independent identically distributed (i.i.d.) complex Gaussian random variables with the mean of zero and the variance of 0.5 per real dimension [11], [39], [40]. The assumption of small-scale Rayleigh flat fading is justified in [11], [39]-[41], i.e., adequate antenna spacing ensures channel decorrelation in a rich-scatting nonline-of-sight radio environment. 
At the $t_{e}$-th energy slot, with antenna selection, the BS selects $M\left(t_{e}\right) \in\{1,2, \cdots, N\}$ antennas with the largest channel gains, i.e., $\sum_{k=1}^{K}\left|h_{k, 1}\right|^{2}>\sum_{k=1}^{K}\left|h_{k, 2}\right|^{2}>\cdots>\sum_{k=1}^{K}\left|h_{k, M\left(t_{e}\right)}\right|^{2}$, from $N$ antennas for data transmission. The rationale behind is that since the contributions of different antennas are not equal, the antennas with largest channel gains should be selected in priority. A similar assumption is also adopted in other previous works [11], [42]. The downlink spectral efficiency (bits/s/Hz) at the $t_{e}$-th energy slot is calculated as [42]

$$
I_{\text {sel }}\left(t_{e}\right)=K \log _{2}\left(1+\frac{P_{T x}\left(t_{e}\right)}{K^{2} \sigma^{2}} \sum_{j=1}^{M\left(t_{e}\right)} \sum_{k=1}^{K}\left|h_{k, j}\right|^{2}\right),
$$

where $P_{T x}\left(t_{e}\right)$ represents the transmission power at the $t_{e}$-th energy slot.

Based on the channel hardening phenomenon [42], we have the following theorem:

Theorem 1. $I_{\text {sel }}$ defined in (4) follows a folded normal distribution, which is expressed as

$$
\begin{aligned}
I_{\text {sel }}\left(t_{e}\right) \sim \mathcal{F N}\left(K \log _{2}\right. & {\left[1+\left(1+\ln \frac{N}{M\left(t_{e}\right)}\right) \frac{P_{T x}\left(t_{e}\right) M\left(t_{e}\right)}{K \sigma^{2}}\right] } \\
& \left.\frac{\left(\log _{2} e P_{T x}\left(t_{e}\right)\right)^{2} M\left(t_{e}\right)\left(2-\frac{M\left(t_{e}\right)}{N}\right)}{K \sigma^{4}\left[1+\left(1+\ln \frac{N}{M\left(t_{e}\right)}\right) \frac{P_{T x} M\left(t_{e}\right)}{K \sigma^{2}}\right]^{2}}\right) .
\end{aligned}
$$

Proof: Please see Appendix A.

Remark 2: $M\left(t_{e}\right)$ represents the number of selected antennas at the $t_{e}$-th energy slot, which is an integer and is less than or equal to $N$. The value of $M\left(t_{e}\right)$ is time-varying and should be dynamically determined by the proposed algorithm. It is noted that $M\left(t_{e}\right)$ only indicates that how many antennas should be selected, while the CSI at each channel slot is used to determine which specific antennas are selected. We assume that the CSI is available for the BS. How to perform CSI estimation is out of the scope of this work and will be studied in the future.

\section{Power Consumption Model}

Let $P_{\text {total }}\left(t_{e}\right)$ denote the total power consumption of the BS at the $t_{e}$-th slot. According to [43], $P_{\text {total }}\left(t_{e}\right)$ is expressed as

$$
P_{\text {total }}\left(t_{e}\right)=P_{C}\left(t_{e}\right)+P_{T x}\left(t_{e}\right)+P_{R F}\left(t_{e}\right) M\left(t_{e}\right),
$$

where $P_{C}\left(t_{e}\right), P_{T x}\left(t_{e}\right)$, and $P_{R F}\left(t_{e}\right)$ represent the instantaneous circuit power, transmission power, and single RF chain circuit power at the $t_{e}$-th slot, respectively. Each part of the power 
consumption comes from both the renewable energy stored in the battery and the power grid. Thus, $P_{C}, P_{T x}$ and $P_{R F}$ are defined as

$$
\begin{gathered}
P_{C}\left(t_{e}\right)=P_{C}^{E}\left(t_{e}\right)+P_{C}^{G}\left(t_{e}\right), \\
P_{T x}\left(t_{e}\right)=P_{T x}^{E}\left(t_{e}\right)+P_{T x}^{G}\left(t_{e}\right), \\
P_{R F}\left(t_{e}\right)=P_{R F}^{E}\left(t_{e}\right)+P_{R F}^{G}\left(t_{e}\right),
\end{gathered}
$$

where $P_{C}^{E}\left(t_{e}\right), P_{T x}^{E}\left(t_{e}\right)$, and $P_{R F}^{E}\left(t_{e}\right)$ represent the instantaneous circuit power, transmission power, and single RF chain circuit power supplied by the battery at the $t_{e}$-th slot, respectively. $P_{C}^{G}\left(t_{e}\right), P_{T x}^{G}\left(t_{e}\right)$, and $P_{R F}^{G}\left(t_{e}\right)$ represent the instantaneous circuit power, transmission power, and single RF circuit chain power supplied by the power grid at the $t_{e}$-th slot, respectively.

The total power consumption of grid power and the total power consumption of renewable energy at the $t_{e}$-th slot are given by

$$
\begin{aligned}
& P_{\text {total }}^{G}\left(t_{e}\right)=P_{C}^{G}\left(t_{e}\right)+P_{T x}^{G}\left(t_{e}\right)+P_{R F}^{G}\left(t_{e}\right) M\left(t_{e}\right), \\
& P_{\text {total }}^{E}\left(t_{e}\right)=P_{C}^{E}\left(t_{e}\right)+P_{T x}^{E}\left(t_{e}\right)+P_{R F}^{E}\left(t_{e}\right) M\left(t_{e}\right) .
\end{aligned}
$$

\section{PROBlEM Formulation}

In this section, we consider the optimization over $T_{\omega}$ price slots, i.e., a total of $T_{e} T_{\omega}$ energy slots. We define the antenna selection and power control policies as $\mathcal{S}=\left\{\mathcal{S}_{1}, \cdots, \mathcal{S}_{t_{e}}, \cdots, \mathcal{S}_{T_{e} T_{\omega}}\right\}$ and $\mathcal{P}=\left\{\mathcal{P}_{1}, \cdots, \mathcal{P}_{t_{e}}, \cdots, \mathcal{P}_{T_{e} T_{\omega}}\right\}$, respectively, where $\mathcal{S}_{t_{e}}=\left\{M\left(t_{e}\right)\right\}$ and $\mathcal{P}_{t_{e}}=\left\{P_{C}^{E}\left(t_{e}\right), P_{C}^{G}\left(t_{e}\right)\right.$, $\left.P_{T x}^{E}\left(t_{e}\right), P_{T x}^{G}\left(t_{e}\right), P_{R F}^{E}\left(t_{e}\right), P_{R F}^{G}\left(t_{e}\right)\right\}$.

The expected throughput per unit bandwidth (bits/Hz) at the $t_{e}$-th slot is given by

$$
U_{s e l}\left(\mathcal{S}_{t_{e}}, \mathcal{P}_{t_{e}}\right)=\mathbb{E}\left\{I_{s e l}\left(t_{e}\right)\right\} T_{0}=K \log _{2}\left[1+\left(1+\ln \frac{N}{M\left(t_{e}\right)}\right) \frac{P_{T x}\left(t_{e}\right) M\left(t_{e}\right)}{K \sigma^{2}}\right] T_{0},
$$

where $\mathbb{E}\{\cdot\}$ denotes the expectation operator.

The energy cost (cents) at the $t_{e}$-th slot is given by

$$
C_{\text {total }}\left(\mathcal{S}_{t_{e}}, \mathcal{P}_{t_{e}}\right)=\omega^{E}\left(t_{e}\right) P_{\text {total }}^{E}\left(t_{e}\right) T_{0}+\omega^{G}\left(t_{e}\right) P_{\text {total }}^{G}\left(t_{e}\right) T_{0} .
$$

Let $\overline{U_{\text {sel }}}(\mathcal{S}, \mathcal{P})$ and $\overline{C_{\text {total }}}(\mathcal{S}, \mathcal{P})$ denote the time-average throughput per unit bandwidth and the time-average energy cost over $T_{e} T_{\omega}$ slots, respectively, which are given by

$$
\begin{aligned}
\overline{U_{\text {sel }}}(\mathcal{S}, \mathcal{P}) & =\lim _{T_{\omega} \rightarrow \infty} \frac{1}{T_{e} T_{\omega}} \sum_{t_{e}=1}^{T_{e} T_{\omega}} \mathbb{E}\left\{U_{\text {sel }}\left(\mathcal{S}_{t_{e}}, \mathcal{P}_{t_{e}}\right)\right\}, \\
\overline{C_{\text {total }}}(\mathcal{S}, \mathcal{P}) & =\lim _{T_{\omega} \rightarrow \infty} \frac{1}{T_{e} T_{\omega}} \sum_{t_{e}=1}^{T_{e} T_{\omega}} \mathbb{E}\left\{C_{\text {total }}\left(\mathcal{S}_{t_{e}}, \mathcal{P}_{t_{e}}\right)\right\}
\end{aligned}
$$


Let $U_{E^{3}}$ denote the energy economic efficiency (bits/Hz/cent) which is defined as the ratio of the time-average throughput per unit bandwidth to the time-average energy cost. $U_{E^{3}}$ can be written as

$$
U_{E^{3}}=\frac{\overline{U_{\text {sel }}}(\mathcal{S}, \mathcal{P})}{\overline{C_{\text {total }}}(\mathcal{S}, \mathcal{P})} .
$$

The energy-economic-efficient joint antenna selection and power control problem is formulated as

$$
\begin{aligned}
& \mathbf{P} 1: \max _{(\mathcal{S}, \mathcal{P})} U_{E^{3}} \\
& \text { s.t. } \quad C_{1} \sim C_{9} \\
& C_{1}: P_{\text {total }}^{E}\left(t_{e}\right) T_{0} \leq B\left(t_{e}\right), \forall t_{e}, \\
& C_{2}: P_{C}^{E}\left(t_{e}\right)+P_{C}^{G}\left(t_{e}\right)=P_{C}\left(t_{e}\right), \forall t_{e}, \\
& C_{3}: P_{R F}^{E}\left(t_{e}\right)+P_{R F}^{G}\left(t_{e}\right)=P_{R F}\left(t_{e}\right), \forall t_{e} . \\
& C_{4}: P_{T x}\left(t_{e}\right) \leq P_{T x, \max }, \forall t_{e}, \\
& C_{5}: P_{\text {total }}^{G}\left(t_{e}\right) \leq P_{\text {max }}^{G}, \forall t_{e}, \\
& C_{6}: U_{\text {sel }}\left(\mathcal{S}_{t_{e}}, \mathcal{P}_{t_{e}}\right) \geq K R_{\text {min }}, \forall t_{e}, \\
& C_{7}: \lim _{T_{\omega} \rightarrow+\infty} \frac{1}{T_{e} T_{\omega}} \sum_{t_{e}=1}^{T_{e} T_{\omega}} \mathbb{E}\left\{P_{T x}\left(t_{e}\right)\right\} \leq P_{T x, \text { mean }}, \\
& C_{8}: M\left(t_{e}\right) \in[1, \ldots, N], \forall t_{e}, \\
& C_{9}: P_{C}^{E}\left(t_{e}\right), P_{C}^{G}\left(t_{e}\right), P_{T x}^{E}\left(t_{e}\right), P_{T x}^{G}\left(t_{e}\right), P_{R F}^{E}\left(t_{e}\right), P_{R F}^{G}\left(t_{e}\right) \geq 0, \forall t_{e},
\end{aligned}
$$

where $C_{1}$ is the energy causality constraint. $C_{2}$ and $C_{3}$ specify that the power required for reliable operations of $\mathrm{BS}$ and single $\mathrm{RF}$ chain should be guaranteed. $C_{4}$ and $C_{5}$ are instantaneous maximum transmission power and grid power constraints. $C_{6}$ is the QoS requirement. $C_{7}$ is the long-term constraint of transmission power. $C_{8}$ and $C_{9}$ specify the boundary constraints of optimization variables.

\section{EnERgy-EConomic-EFficient Online Joint Resource Allocation}

In this section, the proposed joint resource allocation algorithm is presented. First, we introduce how to transform problem $\mathbf{P 1}$ into a sequence of parameterized problems in subtractive form. Then, we introduce the formulation of Lyapunov optimization, in which the long-term stochastic 
optimization problem is converted to a series of short-term deterministic optimization problems. Next, we propose an iterative online joint optimization algorithm based on bisection method and Lagrange dual decomposition.

\section{A. Problem Transformation}

Denote the optimum objective value of $\mathbf{P} 1$ as $q^{*}$, which is given by

$$
q^{*}=\max _{(\mathcal{S}, \mathcal{P})} U_{E^{3}}=\frac{\overline{U_{\text {sel }}}\left(\mathcal{S}^{*}, \mathcal{P}^{*}\right)}{\overline{C_{\text {total }}}\left(\mathcal{S}^{*}, \mathcal{P}^{*}\right)},
$$

where $\mathcal{S}^{*}$ and $\mathcal{P}^{*}$ represent the optimum antenna selection and power control policies, respectively. Based on nonlinear fractional programming [44], we have

Theorem 2. The optimum objective value $q^{*}$ is achievable if and only if

$$
\max _{(\mathcal{S}, \mathcal{P})} \overline{U_{\text {sel }}}(\mathcal{S}, \mathcal{P})-q^{*} \overline{C_{\text {total }}}(\mathcal{S}, \mathcal{P})=\overline{U_{\text {sel }}}\left(\mathcal{S}^{*}, \mathcal{P}^{*}\right)-q^{*} \overline{C_{\text {total }}}\left(\mathcal{S}^{*}, \mathcal{P}^{*}\right)=0
$$

Proof: The details are omitted here due to space limitation. A similar proof can be found in [44].

Based on Theorem 2, there exists an alternative subtractive-form problem which is equivalent to $\mathbf{P} 1$. Thus, $\mathbf{P} 1$ can be rewritten as

$$
\begin{gathered}
\text { P2 } \max _{(\mathcal{S}, \mathcal{P})} \overline{U_{\text {sel }}}(\mathcal{S}, \mathcal{P})-q^{*} \overline{C_{\text {total }}}(\mathcal{S}, \mathcal{P}) \\
\text { s.t. } \quad C_{1} \sim C_{9} .
\end{gathered}
$$

However, the specific value of $q^{*}$ in P2 is still unknown. Therefore, base on [45], we use an alternative variable $q\left(t_{e}\right)$ to replace $q^{*}$, which is defined as

$$
q\left(t_{e}\right)=\frac{\sum_{\tau=1}^{t_{e}} \mathbb{E}\left\{U_{\text {sel }}\left(\mathcal{S}_{\tau}, \mathcal{P}_{\tau}\right)\right\}}{\sum_{\tau=1}^{t_{e}} \mathbb{E}\left\{C_{\text {total }}\left(\mathcal{S}_{\tau}, \mathcal{P}_{\tau}\right)\right\}} .
$$

Then P2 can be rewritten as

$$
\begin{gathered}
\text { P3 }: \max _{(\mathcal{S}, \mathcal{P})} \overline{U_{\text {sel }}}(\mathcal{S}, \mathcal{P})-q\left(t_{e}\right) \overline{C_{\text {total }}}(\mathcal{S}, \mathcal{P}) \\
\text { s.t. } \quad C_{1} \sim C_{9} .
\end{gathered}
$$


Remark 3: P3 is a mixed integer nonlinear programming (MINLP) problem, where both integer and continuous variables have to be jointly optimized. Furthermore, it also involves long-term optimization objective function and constraints. Therefore, P3 is NP-hard and $q\left(t_{e}\right)$ cannot be directly obtained by the conventional Dinkelbach approach [46].

\section{B. The Formulation of Lyapunov Optimization}

Based on the concept of virtual queue [44], the long-term time-average constraint $C_{7}$ of $\mathbf{P 3}$ is converted to a queue stability constraint. Specifically, the virtual queue $Z\left(t_{e}\right)$ associated with $C_{7}$ is given by

$$
Z\left(t_{e}+1\right)=\max \left[Z\left(t_{e}\right)-P_{T x, \text { mean }}, 0\right]+P_{T x}\left(t_{e}\right)
$$

We set the initial queue backlog as $Z(1)=0$.

Theorem 3. If $Z\left(t_{e}\right)$ is mean rate stable, $C_{7}$ holds automatically.

Proof: The detailed proof is provided in Section VI.

Based on Theorem 3, P3 can be rewritten as

$$
\begin{aligned}
\mathbf{P} 4: \max _{(\mathcal{S}, \mathcal{P})} \overline{U_{\text {sel }}}(\mathcal{S}, \mathcal{P})-q\left(t_{e}\right) \overline{C_{\text {total }}}(\mathcal{S}, \mathcal{P}) \\
\text { s.t. } \quad C_{1} \sim C_{6}, C_{8}, C_{9}, \\
C_{10}: \text { Queue } Z \text { is mean rate stable. }
\end{aligned}
$$

Then, the Lyapunov function $L\left(Z\left(t_{e}\right)\right)$ is given by

$$
L\left(Z\left(t_{e}\right)\right)=\frac{1}{2} Z^{2}\left(t_{e}\right)
$$

We introduce a Lyapunov drift to push the Lyapunov function to a lower congestion state and keep the virtual queue stable. The Lyapunov drift is the expected change in the Lyapunov function over one slot, given that the current state at the $t_{e}$-th slot is $Z\left(t_{e}\right)$. Thus, the one-slot conditional Lyapunov drift is defined as

$$
\Delta\left(Z\left(t_{e}\right)\right) \triangleq \mathbb{E}\left\{L\left(Z\left(t_{e}+1\right)\right)-L\left(Z\left(t_{e}\right)\right) \mid Z\left(t_{e}\right)\right\} .
$$

To maximize $\overline{U_{\text {sel }}}(\mathcal{S}, \mathcal{P})-q\left(t_{e}\right) \overline{C_{\text {total }}}(\mathcal{S}, \mathcal{P})$ under the constraint that queue $Z$ is stable, we define the drift-minus-reward term as

$$
D M\left(Z\left(t_{e}\right)\right)=\Delta\left(Z\left(t_{e}\right)\right)-V \mathbb{E}\left\{U_{s e l}\left(\mathcal{S}_{t_{e}}, \mathcal{P}_{t_{e}}\right)-q\left(t_{e}\right) C_{t o t a l}\left(\mathcal{S}_{t_{e}}, \mathcal{P}_{t_{e}}\right) \mid Z\left(t_{e}\right)\right\}
$$


where $V$ is a nonnegative parameter which controls the relative importance of the drift $\Delta\left(Z\left(t_{e}\right)\right)$ compared with the reward $\mathbb{E}\left\{U_{\text {sel }}\left(\mathcal{S}_{t_{e}}, \mathcal{P}_{t_{e}}\right)-q\left(t_{e}\right) C_{\text {total }}\left(\mathcal{S}_{t_{e}}, \mathcal{P}_{t_{e}}\right) \mid Z\left(t_{e}\right)\right\}$, i.e., the tradeoff between "queue stability" and the "reward maximization".

Theorem 4. The drift-minus-reward term, i.e., $\Delta\left(Z\left(t_{e}\right)\right)-V \mathbb{E}\left\{U_{\text {sel }}\left(\mathcal{S}_{t_{e}}, \mathcal{P}_{t_{e}}\right)-q\left(t_{e}\right) C_{t_{\text {total }}}\left(\mathcal{S}_{t_{e}}, \mathcal{P}_{t_{e}}\right)\right.$ $\left.\mid Z\left(t_{e}\right)\right\}$, is upper bounded by

$$
\begin{aligned}
& \Delta\left(Z\left(t_{e}\right)\right)-V \mathbb{E}\left\{U_{\text {sel }}\left(\mathcal{S}_{t_{e}}, \mathcal{P}_{t_{e}}\right)-q\left(t_{e}\right) C_{\text {total }}\left(\mathcal{S}_{t_{e}}, \mathcal{P}_{t_{e}}\right) \mid Z\left(t_{e}\right)\right\} \\
\leq & C+\mathbb{E}\left\{Z\left(t_{e}\right)\left[P_{T x}\left(t_{e}\right)-P_{T x, \text { mean }}\right] \mid Z\left(t_{e}\right)\right\}-V \mathbb{E}\left\{U_{\text {sel }}\left(\mathcal{S}_{t_{e}}, \mathcal{P}_{t_{e}}\right)-q\left(t_{e}\right) C_{\text {total }}\left(\mathcal{S}_{t_{e}}, \mathcal{P}_{t_{e}}\right) \mid Z\left(t_{e}\right)\right\}
\end{aligned}
$$

where $C$ is a finite positive constant that satisfies

$$
C \geq \mathbb{E}\left\{\frac{P_{T x}^{2}\left(t_{e}\right)+P_{T x, \text { mean }}^{2}}{2} \mid Z\left(t_{e}\right)\right\} .
$$

Proof: The detailed proof is omitted here due to space limitation. A similar proof can be found in [35].

According to the principle of Lyapunov optimization, the upper bound of the drift-minusreward term defined in (26) is minimized at each slot subject to constraints $C_{1} \sim C_{6}, C_{8}$, and $C_{9}$. Thus, $\mathbf{P 4}$ can be rewritten as

$$
\begin{aligned}
& \text { P5 : } \min _{\left(\mathcal{S}_{t_{e}}, \mathcal{P}_{t_{e}}\right)} Z\left(t_{e}\right)\left[P_{T x}\left(t_{e}\right)-P_{T x, \text { mean }}\right]-V\left[U_{\text {sel }}\left(\mathcal{S}_{t_{e}}, \mathcal{P}_{t_{e}}\right)-q\left(t_{e}\right) C_{\text {total }}\left(\mathcal{S}_{t_{e}}, \mathcal{P}_{t_{e}}\right)\right] \\
& \quad \text { s.t. } \quad C_{1} \sim C_{6}, C_{8}, C_{9} .
\end{aligned}
$$

\section{Joint Antenna Selection and Power Control}

In this subsection, we propose an iterative online algorithm to solve P5, which consists of the antenna selection algorithm summarized in Algorithm 1, and the power control algorithm summarized in Algorithm 2.

1) Antenna Selection: Algorithm 1 is based on the bisection method [47], which is an effective low-complexity searching algorithm with logarithmic complexity. It has been widely employed in various application scenarios including antenna selection [11] and power allocation [48]. It is noted that the bisection-based antenna selection algorithm developed in [11] cannot be directly applied because the coupling between antenna selection and energy domain optimization is not considered. Let $\lfloor x\rfloor$ denote the largest integer that is less than or equal to $x$, and $\lceil x\rceil$ 




denote the smallest integer that is not less than $x$. At the $t_{e}$-th slot, we start with an interval $\left[M_{\text {low }}\left(t_{e}\right), M_{\text {high }}\left(t_{e}\right)\right]$, which is known to contain the optimum antenna number $M^{*}\left(t_{e}\right)$. In each iteration, we calculate $M_{m i d}\left(t_{e}\right)$ and $M_{m i d}\left(t_{e}\right)-1$, and obtain the corresponding optimum power control policy $\hat{\mathcal{P}}_{t_{e}}^{\prime}$ and $\hat{\mathcal{P}}_{t_{e}}^{\prime \prime}$ respectively by using Algorithm 2 . It is noted that only the last two terms of P5 involve the variables of antenna selection. Thus, we define $F_{1}\left(M\left(t_{e}\right), \mathcal{P}_{t_{e}},\left(t_{e}\right)\right)$ as

$$
F_{1}\left(M\left(t_{e}\right), \mathcal{P}_{t_{e}}, q\left(t_{e}\right)\right)=U_{\text {sel }}\left(M\left(t_{e}\right), \mathcal{P}_{t_{e}}\right)-q\left(t_{e}\right) C_{\text {total }}\left(M\left(t_{e}\right), \mathcal{P}_{t_{e}}\right)
$$

Then, we compare $F_{1}\left(M_{m i d}\left(t_{e}\right), \hat{\mathcal{P}}_{t_{e}}^{\prime}, \hat{q}^{\prime}\left(t_{e}\right)\right)$ with $F_{1}\left(M_{\text {mid }}\left(t_{e}\right)-1, \hat{\mathcal{P}}_{t_{e}}^{\prime \prime}, \hat{q}^{\prime \prime}\left(t_{e}\right)\right)$ to determine whether $M^{*}\left(t_{e}\right)$ lies in the interval $\left[M_{l o w}\left(t_{e}\right), M_{m i d}\left(t_{e}\right)\right]$ or the interval $\left[M_{m i d}\left(t_{e}\right), M_{h i g h}\left(t_{e}\right)\right]$, 


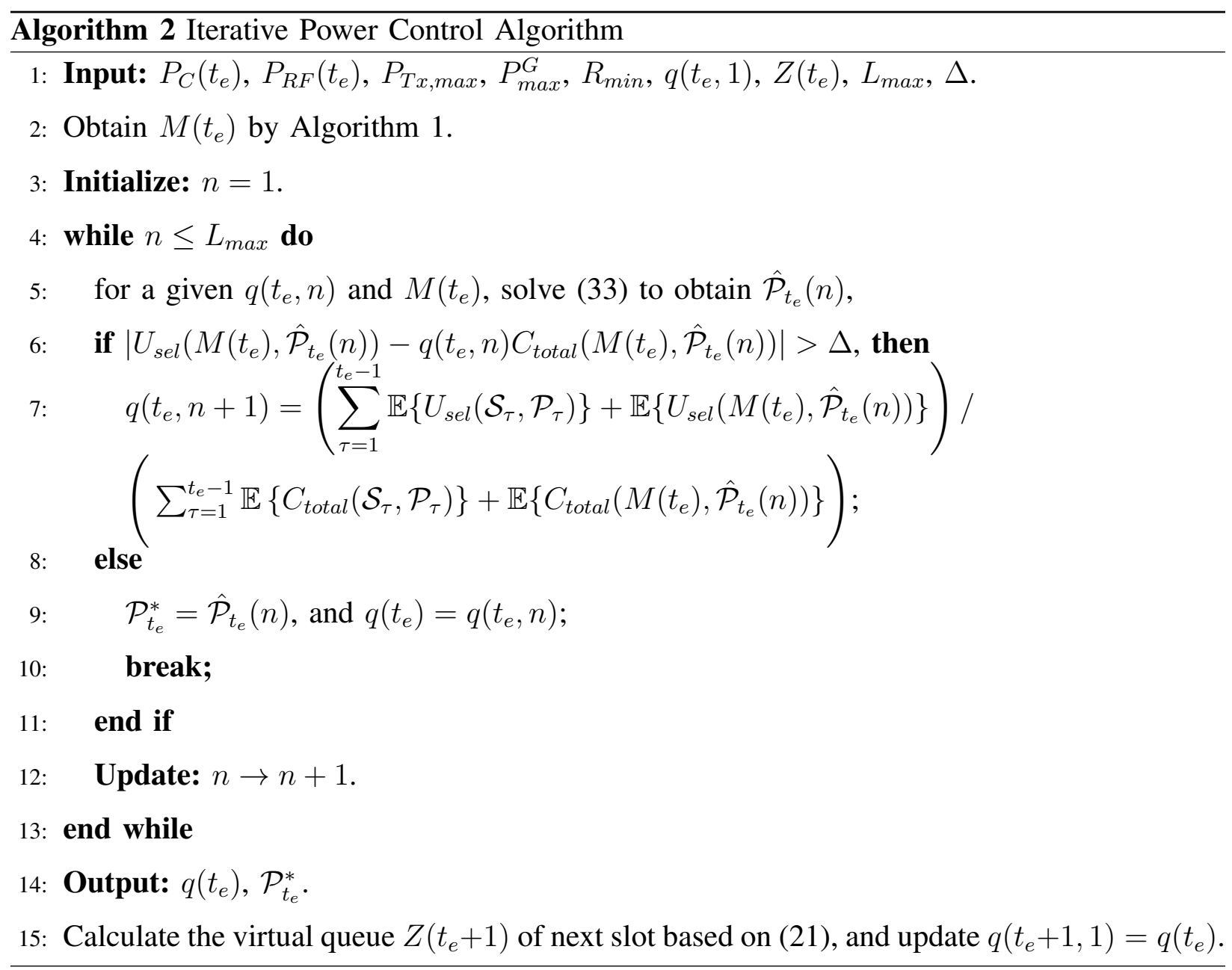

which is given by

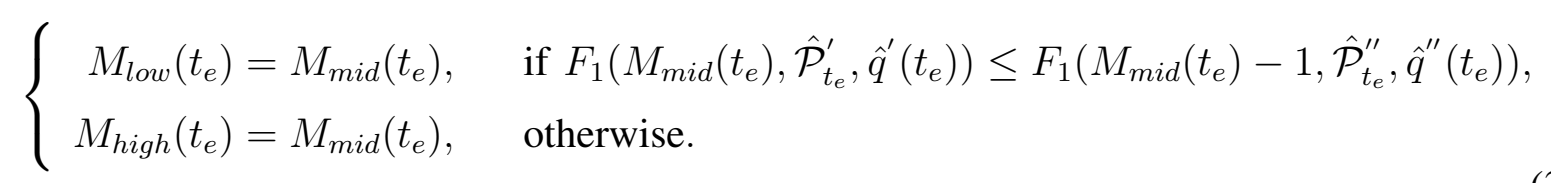

If $F_{1}\left(M_{\text {mid }}\left(t_{e}\right), \hat{\mathcal{P}}_{t_{e}}^{\prime}, \hat{q}^{\prime}\left(t_{e}\right)\right) \leq F_{1}\left(M_{\text {mid }}\left(t_{e}\right)-1, \hat{\mathcal{P}}_{t_{e}}^{\prime \prime}, \hat{q}^{\prime \prime}\left(t_{e}\right)\right), M^{*}\left(t_{e}\right)$ lies in the interval $\left[M_{\text {mid }}\left(t_{e}\right)\right.$, $\left.M_{\text {high }}\left(t_{e}\right)\right]$ and $M_{\text {low }}\left(t_{e}\right)=M_{\text {mid }}\left(t_{e}\right)$. Otherwise, $M^{*}\left(t_{e}\right)$ lies in the interval $\left[M_{\text {low }}\left(t_{e}\right), M_{\text {mid }}\left(t_{e}\right)\right]$ and $M_{\text {high }}\left(t_{e}\right)=M_{\text {mid }}\left(t_{e}\right)$. The details are provided in line $5 \sim 11$ of Algorithm 1 .

2) Power Control: The power control algorithm is provided as Algorithm 2. $L_{\max }$ denotes the total iteration number, $n$ denotes the iteration index, and $\Delta$ denotes the tolerance threshold. We use $q\left(t_{e}, n\right)$ to represent $q\left(t_{e}\right)$ obtained from the $(n-1)$-th iteration at the $t_{e}$-th slot, the initial value of which is $q(1,1)=0$. Then, at the $n$-th iteration, by using $q\left(t_{e}, n\right)$, we can obtain 
$\hat{\mathcal{P}}_{t_{e}}(n)$ associated with $M\left(t_{e}\right)$ by solving the following power control problem.

$$
\begin{aligned}
& \text { P6 }: \min _{\left(\mathcal{P}_{e}\right)} Z\left(t_{e}\right)\left[P_{T x}\left(t_{e}\right)-P_{T x, \text { mean }}\right]-V\left[U_{\text {sel }}\left(M\left(t_{e}\right), \mathcal{P}_{t_{e}}(n)\right)-q\left(t_{e}, n\right) C_{\text {total }}\left(M\left(t_{e}\right), \mathcal{P}_{t_{e}}(n)\right)\right] \\
& \text { s.t. } \quad C_{1} \sim C_{6}, C_{9} .
\end{aligned}
$$

It is noted that (31) is concave with differentiable objective function and constraints. The corresponding augmented Lagrangian is give by (32).

$$
\begin{aligned}
& \mathcal{L}_{M\left(t_{e}\right)}^{E^{3}}\left(\mathcal{P}_{t_{e}}, \alpha_{t_{e}}, \beta_{t_{e}}, \gamma_{t_{e}}, \delta_{t_{e}}, \zeta_{t_{e}}, \theta_{t_{e}}\right)=Z\left(t_{e}\right)\left[P_{T x}\left(t_{e}\right)-P_{T x, \text { mean }}\right] \\
- & V\left[U_{\text {sel }}\left(M\left(t_{e}\right), \mathcal{P}_{t_{e}}\right)-q\left(t_{e}, n\right) C_{\text {total }}\left(M\left(t_{e}\right), \mathcal{P}_{t_{e}}(n)\right)\right]+\alpha_{t_{e}}(n)\left[P_{\text {total }}^{E}\left(t_{e}\right) T_{0}-B\left(t_{e}\right)\right] \\
+ & \beta_{t_{e}}(n)\left[P_{C}^{E}\left(t_{e}\right)+P_{C}^{G}\left(t_{e}\right)-P_{C}\left(t_{e}\right)\right]+\gamma_{t_{e}}(n)\left[P_{R F}^{E}\left(t_{e}\right)+P_{R F}^{G}\left(t_{e}\right)-P_{R F}\left(t_{e}\right)\right] \\
+ & \delta_{t_{e}}(n)\left[P_{T x}^{E}\left(t_{e}\right)+P_{T x}^{G}\left(t_{e}\right)-P_{T x, \text { mean }}\right]+\zeta_{t_{e}}(n)\left[P_{\text {total }}^{G}\left(t_{e}\right)-P_{\text {max }}^{G}\right] \\
- & \theta_{t_{e}}(n)\left[U_{\text {sel }}\left(M\left(t_{e}\right), \mathcal{P}_{t_{e}}\right)-K R_{\text {min }}\right] .
\end{aligned}
$$

The Lagrange multipliers associated with constraints $C_{1} \sim C_{6}$ are defined as $\alpha_{t_{e}}, \beta_{t_{e}}, \gamma_{t_{e}}, \delta_{t_{e}}, \zeta_{t_{e}}$, and $\theta_{t_{e}}$, respectively. The equivalent Lagrange dual problem is given by

$$
\max _{\left(\alpha_{t_{e}}, \beta_{t_{e}}, \gamma_{t_{e}}, \delta_{t_{e}}, \zeta_{t_{e}}, \theta_{t_{e}} \geq 0\right)} \min _{\left(\mathcal{P}_{t_{e}}\right)} \mathcal{L}_{M\left(t_{e}\right)}^{E^{3}}\left(\mathcal{P}_{t_{e}}, \alpha_{t_{e}}, \beta_{t_{e}}, \gamma_{t_{e}}, \delta_{t_{e}}, \zeta_{t_{e}}, \theta_{t_{e}}\right)
$$

Denote the optimal power control policy associated with $q\left(t_{e}, n\right)$ as $\hat{\mathcal{P}}_{t_{e}}(n)=\left\{\hat{\mathcal{P}}_{C}^{E}\left(t_{e}, n\right)\right.$, $\left.\hat{\mathcal{P}}_{C}^{G}\left(t_{e}, n\right), \hat{\mathcal{P}}_{T x}^{E}\left(t_{e}, n\right), \hat{\mathcal{P}}_{T x}^{G}\left(t_{e}, n\right), \hat{\mathcal{P}}_{R F}^{E}\left(t_{e}, n\right), \hat{\mathcal{P}}_{R F}^{G}\left(t_{e}, n\right)\right\} . \hat{\mathcal{P}}_{t_{e}}(n)$ can be obtained by using KarushKuhn-Tucker (KKT) conditions. Then, the Lagrange multipliers are updated based on the subgradient method [49], [50]. Next, $q\left(t_{e}, n+1\right)$ for the next iteration is calculated based on $M\left(t_{e}\right)$ and $\hat{\mathcal{P}}_{t_{e}}(n)$. The details are provided in line $4 \sim 12$ of Algorithm 2 .

\section{PERFormanCE ANALysis}

In this section, the theoretical properties in terms of queue stability, performance bound, and complexity are analyzed.

\section{A. Stability of Virtual Queue}

If the virtual queue $Z\left(t_{e}\right)$ is mean rate stable, then the constraint $C_{7}$ holds automatically. The proof is provided as follows.

Theorem 5. The virtual queue $Z\left(t_{e}\right)$ is mean rate stable. 
TABLE I

Simulation PARAMETERs.

\begin{tabular}{|l|l|}
\hline Parameter & Value \\
\hline Maximum transmission power constraint $P_{T x, \text { max }}$ & $46 \mathrm{dBm}$ \\
\hline Average transmission power constraint $P_{T x, \text { mean }}$ & $45 \mathrm{dBm}$ \\
\hline Constant operation power $P_{C}$ & $160.8 \mathrm{~W}$ \\
\hline RF chain power $P_{R F}$ & $0,160,450 \mathrm{~mW}$ \\
\hline Antenna number $N$ & 100 \\
\hline Maximum grid power $P_{\max }^{G}$ & $300 \mathrm{~W}$ \\
\hline QoS $R_{\text {min }}$ & $7 \mathrm{bits} / \mathrm{Hz}$ \\
\hline The number of smart terminals $K$ & 4 \\
\hline The variance $\sigma_{0}^{2}$ & 1 \\
\hline
\end{tabular}

Proof: We assume that both $U_{\text {sel }}\left(\mathcal{S}_{t_{e}}, \mathcal{P}_{t_{e}}\right)$ and $C_{\text {total }}\left(\mathcal{S}_{t_{e}}, \mathcal{P}_{t_{e}}\right)$ are bounded by

$$
\begin{gathered}
U_{\text {sel,min }} \leq U_{\text {sel }}\left(\mathcal{S}_{t_{e}}, \mathcal{P}_{t_{e}}\right) \leq U_{\text {sel,max }} \\
C_{\text {total,min }} \leq C_{\text {total }}\left(\mathcal{S}_{t_{e}}, \mathcal{P}_{r}\right) \leq C_{\text {total,max }}
\end{gathered}
$$

where $U_{\text {sel,min }}, U_{\text {sel,max }}, C_{\text {total,min }}$, and $C_{\text {total,max }}$ are finite positive constants. Based on (26) and (34), there exists a positive constant $C$ that satisfies

$$
\Delta\left(Z\left(t_{e}\right)\right) \leq C
$$

Then, according to (24), we have

$$
\mathbb{E}\left\{L\left(Z\left(t_{e}+1\right)\right)-L\left(Z\left(t_{e}\right)\right) \mid Z\left(t_{e}\right)\right\} \leq C .
$$

We can take expectations of both sides of (36) and use the law of iterated expectations to yield

$$
\mathbb{E}\left\{L\left(Z\left(t_{e}+1\right)\right)\right\}-\mathbb{E}\left\{L\left(Z\left(t_{e}\right)\right)\right\} \leq C
$$

Summing over $t_{e} \in\left\{1,2, \cdots, T_{e} T_{\omega}\right\}$ and using the law of telescoping sums yields

$$
\mathbb{E}\left\{L\left(Z\left(T_{e} T_{\omega}\right)\right)\right\}-\mathbb{E}\{L(Z(1))\} \leq C T_{e} T_{\omega}
$$

Using (23), (38) can be rewritten as

$$
\mathbb{E}\left\{Z\left(T_{e} T_{\omega}\right)^{2}\right\} \leq 2 C T_{e} T_{\omega}+2 \mathbb{E}\{L(Z(1))\}
$$


Since the variance of $\left|Z\left(T_{e} T_{\omega}\right)\right|$ is positive, we have $\mathbb{E}\left\{Z\left(T_{e} T_{\omega}\right)^{2}\right\} \geq \mathbb{E}\left\{Z\left(T_{e} T_{\omega}\right)\right\}^{2}$. Thus, we can get

$$
\mathbb{E}\left\{Z\left(T_{e} T_{\omega}\right)\right\} \leq \sqrt{2 C T_{e} T_{\omega}+2 \mathbb{E}\{L(Z(1))\}} .
$$

Next, dividing (40) by $T_{e} T_{\omega}$ and taking the limit $T_{\omega} \rightarrow \infty$ proves that

$$
\lim _{T_{\omega} \rightarrow \infty} \frac{\mathbb{E}\left\{Z\left(T_{e} T_{\omega}\right)\right\}}{T_{e} T_{\omega}}=0 .
$$

Thus, the virtual queue $Z\left(t_{e}\right)$ is mean rate stable.

We can rewrite $(21)$ as

$$
Z\left(t_{e}+1\right)-Z\left(t_{e}\right)=P_{T x}\left(t_{e}\right)-\hat{P}\left(t_{e}\right)
$$

where $\hat{P}\left(t_{e}\right)$ is given by

$$
\hat{P}\left(t_{e}\right)=\min \left[Z\left(t_{e}\right), P_{T x, \text { mean }}\right] .
$$

Summing (42) over $t_{e}=\left[1,2, \cdots, T_{e} T_{\omega}\right]$ and using the law of telescoping sums yields:

$$
Z\left(T_{e} T_{\omega}\right)-Z(1)=\sum_{t_{e}=1}^{T_{e} T_{\omega}} P_{T x}\left(t_{e}\right)-\sum_{t_{e}=1}^{T_{e} T_{\omega}} \hat{P}\left(t_{e}\right) .
$$

Combining (43) and (44), we have

$$
Z\left(T_{e} T_{\omega}\right)-Z(1) \geq \sum_{t_{e}=1}^{T_{e} T_{\omega}} P_{T x}\left(t_{e}\right)-\sum_{t_{e}=1}^{T_{e} T_{\omega}} P_{T x, \text { mean }} .
$$

Finally, dividing (45) by $T_{e} T_{\omega}$ and taking the limit $T_{\omega} \rightarrow \infty$, we have

$$
\lim _{T_{\omega} \rightarrow+\infty} \frac{1}{T_{e} T_{\omega}} \sum_{t_{e}=1}^{T_{e} T_{\omega}} \mathbb{E}\left\{P_{T x}\left(t_{e}\right)\right\} \leq P_{T x, \text { mean }}
$$

This proves that constraint $C_{7}$ holds.

\section{B. Tradeoff between Queue Stability and Reward Maximization}

The energy economic efficiency performance and the average queue backlog performance obtained by the proposed algorithm is given in Theorem 6 .

Theorem 6. The energy economic efficiency $U_{E^{3}}$ and the average backlog of the virtual queue $Z$ are bounded by

$$
\begin{aligned}
U_{E^{3}} & \geq q^{*}-\frac{C}{V C_{t o t a l, \min }}, \\
Z & \leq \frac{C+V\left(U_{\text {sel,max }}-q^{*} C_{t o t a l, \text { min }}\right)}{\epsilon} .
\end{aligned}
$$


Proof: Please see Appendix B.

Remark 4: Theorem 6 shows that economic energy efficiency achieved by the algorithm is bounded as

$$
q^{*}-\frac{C}{V C_{\text {total,min }}} \leq U_{E^{3}} \leq q^{*} .
$$

When $V$ is large enough, the value of $\frac{C}{V C_{t o t a l, m i n}}$ can be arbitrarily small, so that $U_{E^{3}}$ can arbitrarily approach the optimum objective value $q^{*}$. As a result, there exists an $[O(1 / V), O(V)]$ tradeoff between "queue stability" and "reward maximization".

\section{Complexity Analysis}

For each slot, Algorithm 1 requires at most $\left\lceil\log _{2}\left[M_{\text {high }}\left(t_{e}\right)-M_{l o w}\left(t_{e}\right)\right]\right\rceil+1$ comparisons to find the optimum $M^{*}\left(t_{e}\right)$. In comparison, the computational complexity of the exhaustive search method grows exponentially as $N$ increases. For example, with a total of $N=100$ antennas, Algorithm 1 needs approximately 8 comparisons to find the optimal number of selected antennas while the exhaustive search method requires 99 comparisons, i.e., the computational complexity is reduced by nearly $98.9 \%$. Hence, Algorithm 1 has a much lower complexity than the exhaustive method. Algorithm 2 has a complexity $\mathcal{O}\left(L_{\text {max }}^{\text {Loop }} \times L_{\text {max }}^{\text {dual }}\right)$, where $L_{\text {max }}^{\text {Loop }}$ and $L_{\text {max }}^{\text {dual }}$ are iteration numbers needed by the nonlinear fractional programming and Lagrange dual decomposition in each slot, respectively.

\section{Simulation Results}

In this section, we verify the proposed algorithm through simulations. Simulation parameters are summarized in Table I [33], [43], [51]. Two heuristic algorithms are used as baselines for comparison purpose. The baseline 1 algorithm relies on the statistical information of energy arrival and electricity price for decision making and always uses all the available antennas, i.e., antenna selection is not considered. The baseline 2 algorithm is developed based on the snapshotbased optimal algorithm [52], which maximizes $U_{E^{3}}$ at each energy slot without considering the long-term optimization objective and constraints.

Fig. 20 shows the relations between the energy economic efficiency $U_{E^{3}}$ and the selected antenna number $M$ in one energy slot. The electricity prices of renewable energy and grid power are set as $\omega^{E}=0.3$ cents $/ \mathrm{kWh}$ and $\omega^{G}=3$ cents $/ \mathrm{kWh}$, respectively. For the purpose of illustration, the battery capacity is set as $B_{\max }=1000 \mathrm{~J}$, and the energy arrival is set as 


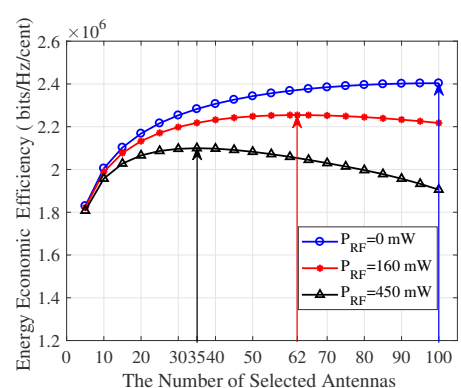

Fig. 2. Energy economic efficiency $U_{E^{3}}$ versus the number of selected antennas $M$.

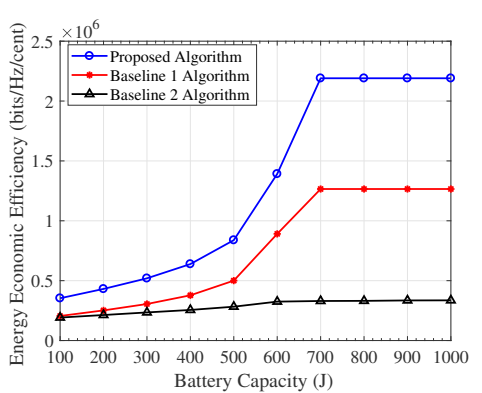

Fig. 3. Energy economic efficiency $U_{E^{3}}$ versus the battery capacity $B_{\max }$.

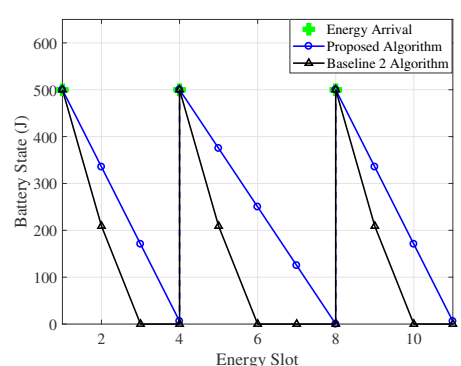

Fig. 4. A snapshot of the battery state.

$E_{i n}\left(t_{e}\right)=700 \mathrm{~J}$. Simulation results demonstrate that $U_{E^{3}}$ is a monotonically increasing function of $M$ in the ideal case that the single RF chain is energy free, i.e., $P_{R F}=0 \mathrm{~mW}$. However, in the nonideal case, i.e., $P_{R F}=160 \mathrm{~mW}$ or $P_{R F}=450 \mathrm{~mW}, U_{E^{3}}$ increases first and then decreases monotonically with $M$. The optimum antenna numbers for $P_{R F}=160 \mathrm{~mW}$ or $P_{R F}=450 \mathrm{~mW}$ are $M^{*}=62$ and $M^{*}=35$, respectively. We found that the optimum antenna number decreases monotonically as $P_{R F}$ increases.

Fig. 12 shows the relations between the energy economic efficiency $U_{E^{3}}$ and the battery capacity $B_{\max }$. We set $T_{\omega}=20$ and $T_{e}=5$, i.e., a total of 100 energy slots. The energy slot duration is set as $T_{0}=3 \mathrm{~s}[35,52,53]$. It is noted that the proposed algorithm is also applicable to other timescales, which provides great scalability and compatibility. The parameter $V$ is set as 10 , and $E_{i n}\left(t_{e}\right)$ follows a uniform distribution within the interval $[0,500] \mathrm{J}$. The entire region can be divided into two regimes, i.e., the battery-capacity limited regime $\left(0 \mathrm{~J} \leq B_{\max } \leq 600 \mathrm{~J}\right)$, and the renewable-energy limited regime (600 $\left.\mathrm{J} \leq B_{\max } \leq 1000 \mathrm{~J}\right)$. In the battery-capacity limited regime, the performance gap between the proposed algorithm and baseline algorithms is small because the performance is degraded significantly due to the frequent energy overflow caused by the limited battery capacity. On the other hand, in the renewable-energy limited regime, the proposed algorithm outperforms the baseline 1 algorithm and baseline 2 algorithm by $73 \%$ and $552 \%\left(B_{\max }=1000 \mathrm{~J}\right)$, respectively. The reason is further elaborated in Fig. 21 and Fig. 22. The baseline 2 algorithm without the awareness of long-term optimization objective and constraints performs the worst among the three.

The snapshots of battery states and energy economic efficiency are shown in Fig. 21 and Fig. 22, respectively. We consider $T_{\omega}=2$, and $T_{e}=5$, i.e., a total of $T_{e} T_{\omega}=10$ energy slots. The 


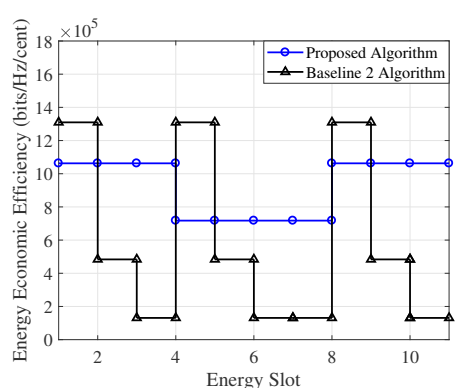

Fig. 5. A snapshot of the energy economic efficiency $U_{E^{3}}$.

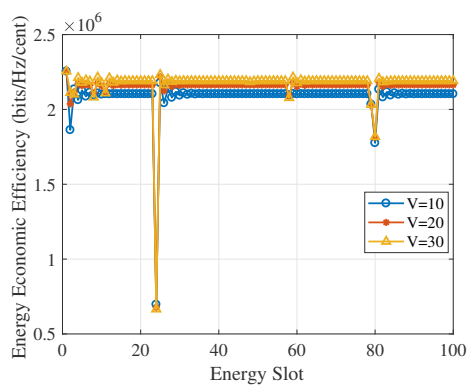

Fig. 8. A snapshot of energy economic efficiency.

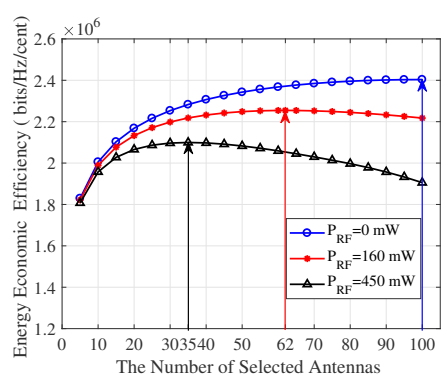

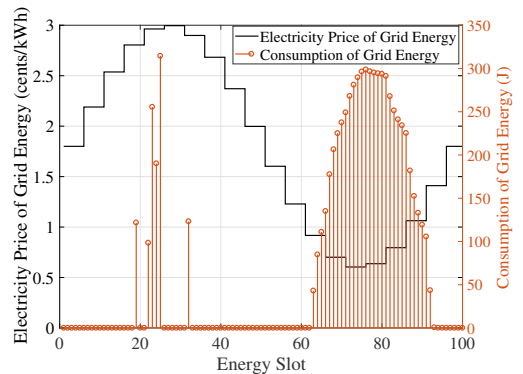

Fig. 6. Adaptiveness for the electricity price of grid energy.

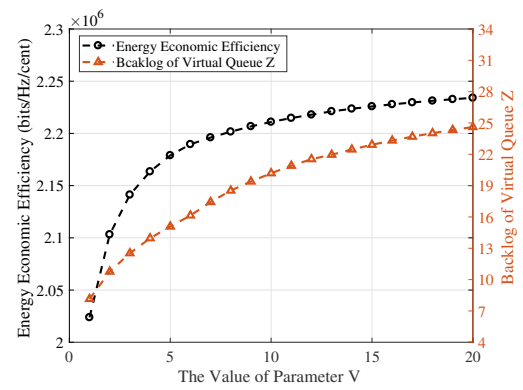

Fig. 9. Energy economic efficiency performance and backlog of queue $Z$ versus the parameter $V$.

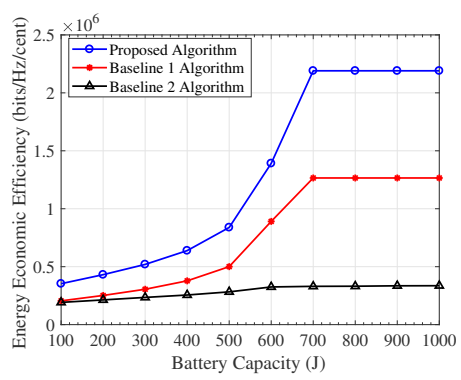

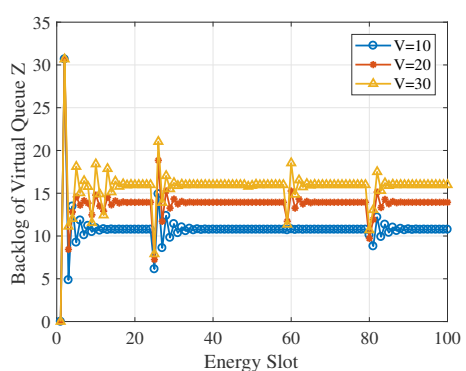

Fig. 7. A snapshot of the backlog of virtual queue $Z$.

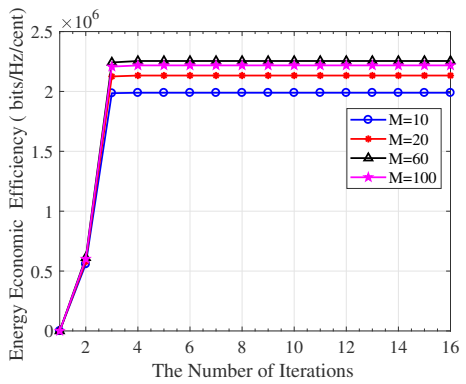

Fig. 10. The convergence performances with different value of $M$.

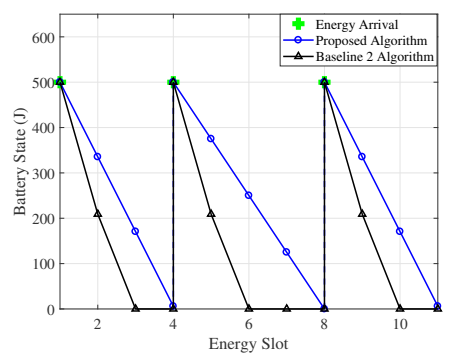

Fig. 11. Economic energy efficiency Fig. 12. Economic energy Efficiency Fig. 13. A snapshot of the battery $U_{E^{3}}$ versus the number of selected $U_{E^{3}}$ versus the battery capacity $B_{\max }$. state. antennas $M$.

slot duration is set as $T_{0}=1 \mathrm{~s}$. The battery capacity is set as $B_{\max }=700 \mathrm{~J}$, and the initial battery state is $500 \mathrm{~J}$. The parameter $V$ is set as 10 . The energy arrival is set as $E_{i n}\left(t_{e}\right)=500 \mathrm{~J}$ when $t_{e}=4,8$, and $E_{i n}\left(t_{e}\right)=0 \mathrm{~J}$ otherwise. In the baseline 2 algorithm, the renewable energy is 

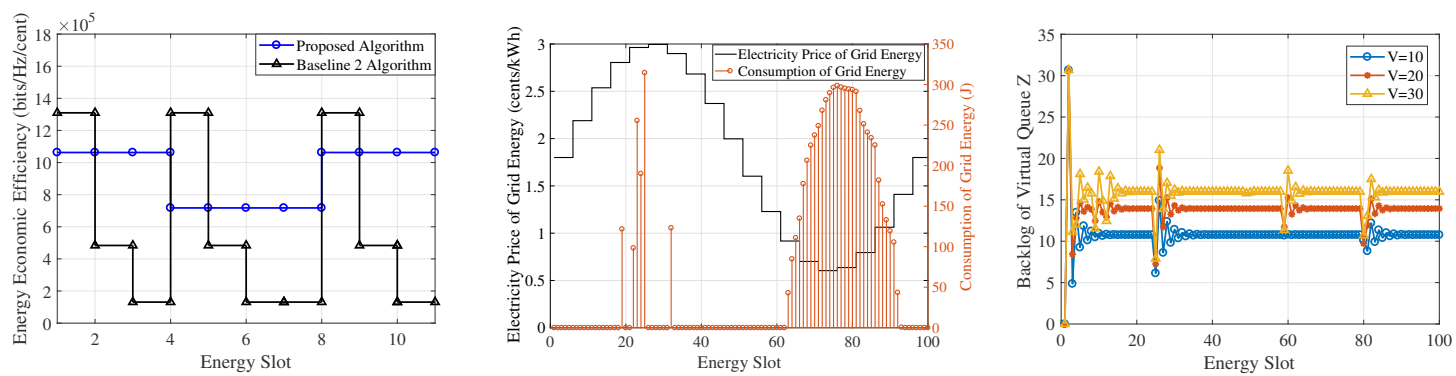

Fig. 14. A snapshot of the energy Fig. 15. Consumption of grid power Fig. 16. Virtual queue backlog versus

economic efficiency $U_{E^{3}}$.

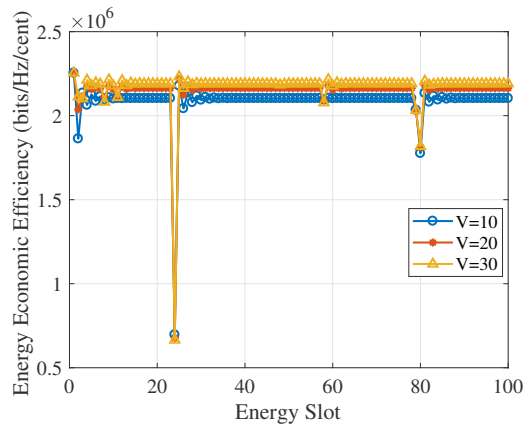

Fig. 17. Energy economic efficiency versus control parameter $V$. versus electricity price.

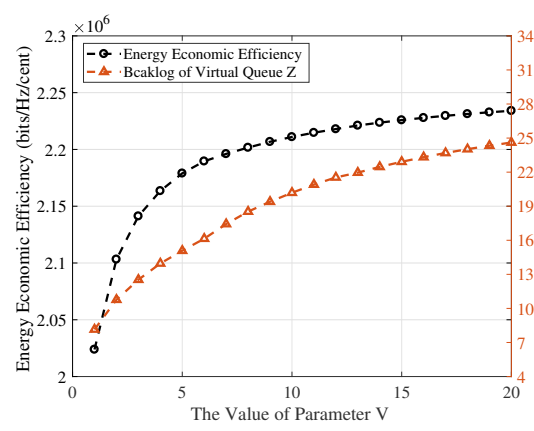

Fig. 18. The tradeoff between energy economic efficiency and virtual queue backlog.

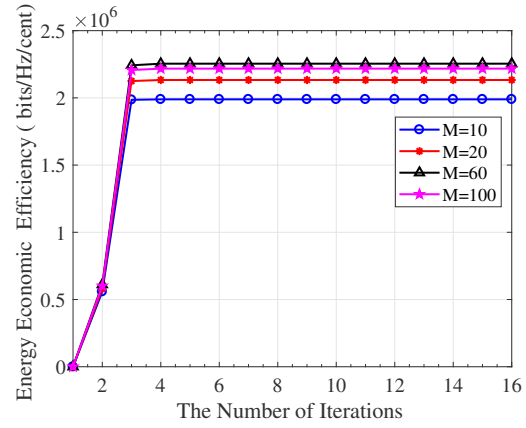

Fig. 19. Energy economic efficiency versus number of iterations $n$.

used more aggressively than the proposed algorithm because only the performance of the current slot is optimized. For example, the baseline 2 algorithm uses out all the stored energy at the 6-th slot while the next energy arrival is at the 8-th slot. Then, the expensive grid power has to be used, which results in severe performance degradation as shown in Fig. 22.

Fig. 23 shows relations between electricity price and energy consumption. We set $T_{\omega}=20$ and $T_{e}=5$, i.e., a total of $T_{e} T_{\omega}=100$ energy slots. The parameter $V$ is set as $10 . E_{i n}\left(t_{e}\right)$ follows a uniform distribution within the interval $[0,700] \mathrm{J}$ when $t_{e}=[1,50]$ slots, and follows a uniform distribution within the interval $[0,400] \mathrm{J}$ when $t_{e}=[51,100]$ slots. The price of renewable energy is set as 1 cent $/ \mathrm{kWh}$. Simulation results show that the proposed algorithm can dynamically adapt grid power consumption with time-varying electricity price. It is able to reduce the consumption amount of the expensive grid power during the peak-price period.

Fig. 24 and Fig. 25 show the impact of control parameter $V$ on queue backlog and energy 


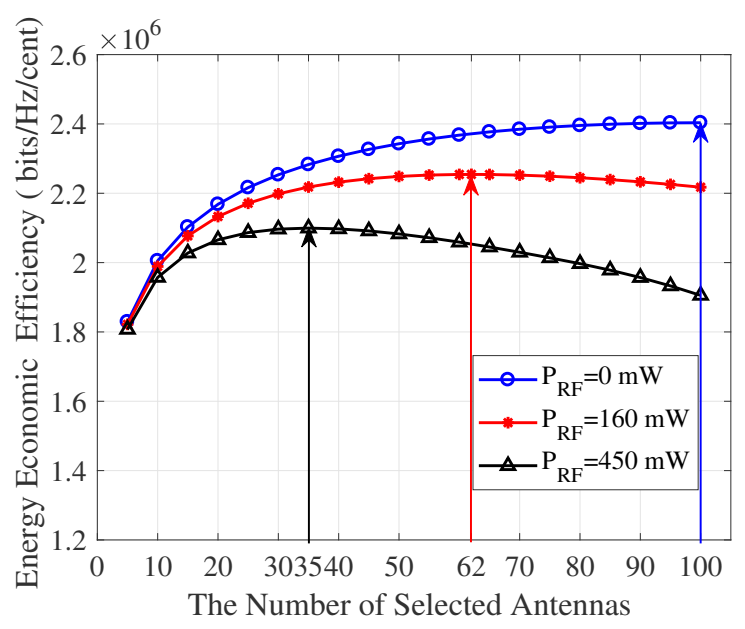

Fig. 20. Economic energy efficiency $U_{E^{3}}$ versus the number of selected antennas $M$.

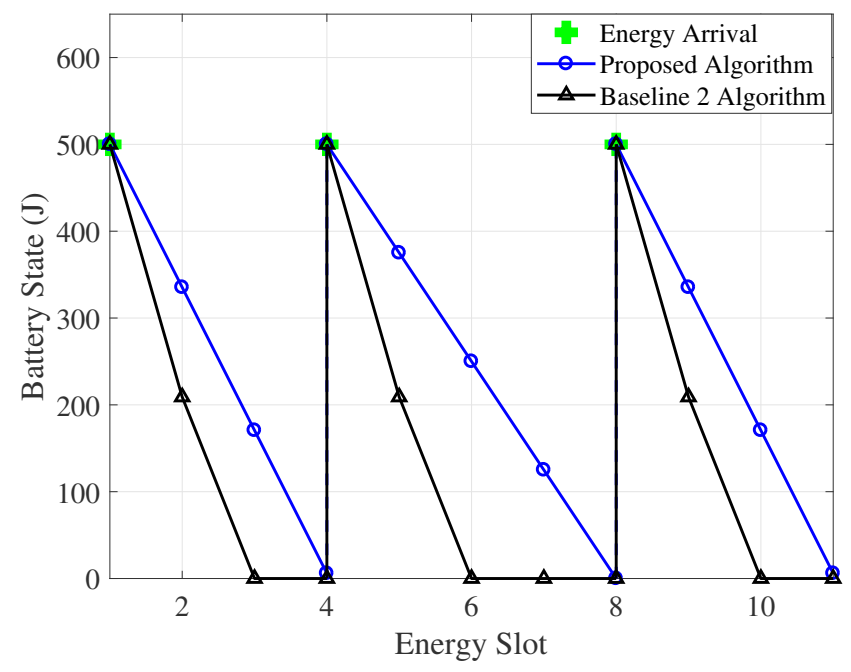

Fig. 21. A snapshot of the battery state.

economic efficiency, respectively. We compare the performances under three scenarios that $V=$ 10, 20,30. $E_{i n}\left(t_{e}\right)$ follows a uniform distribution within the interval $[0,700]$ J. Fig. 24 shows that the queue backlog is able to converge to a stable state within a short period of time under all the scenarios. The change of $V$ mainly affects the final stable state rather than the convergence speed. Fig. 25 shows that the energy economic efficiency of the proposed algorithm is dynamically optimized in accordance with the energy arrival and electricity prices. The $[O(1 / V), O(V)]$ 


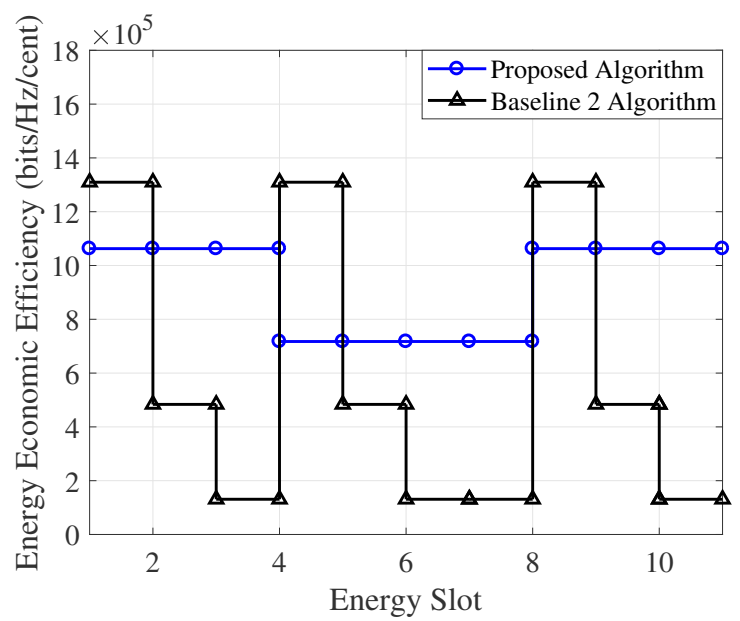

Fig. 22. A snapshot of the energy economic efficiency.

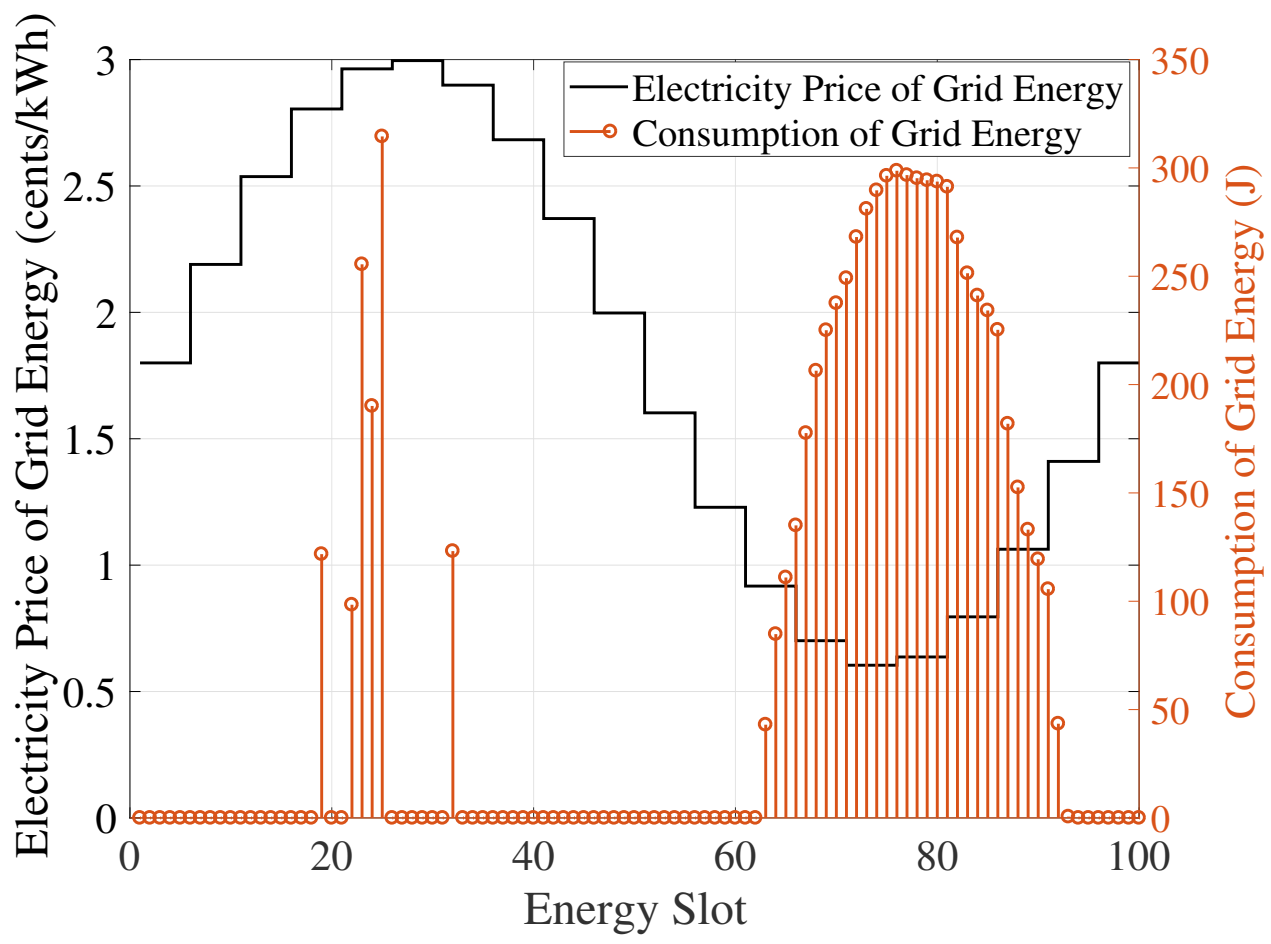

Fig. 23. Adaptiveness for the electricity price of smart grid.

tradeoff between queue backlog and energy economic efficiency is also validated in Fig. 26, i.e., the energy economic efficiency decreases monotonically with $V$ while the queue backlog increases monotonically with $V$. 


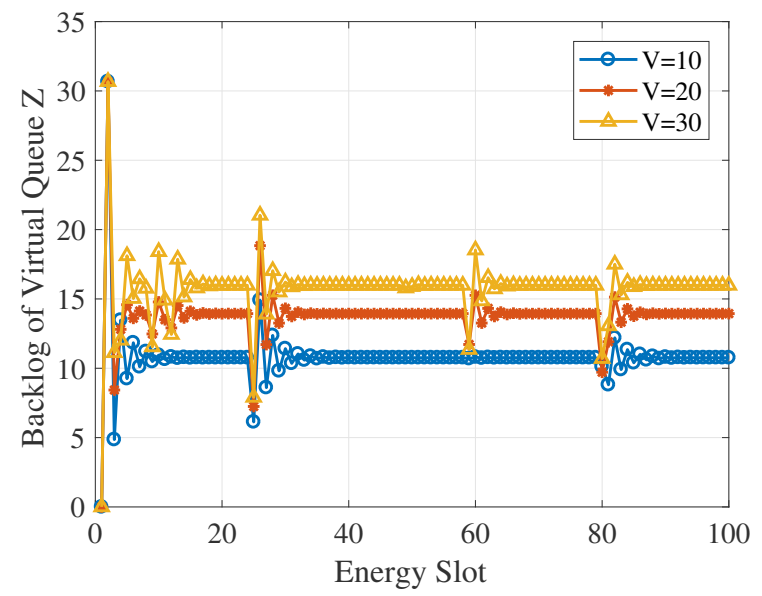

Fig. 24. A snapshot of the backlog of virtual queue $\mathrm{Z}$

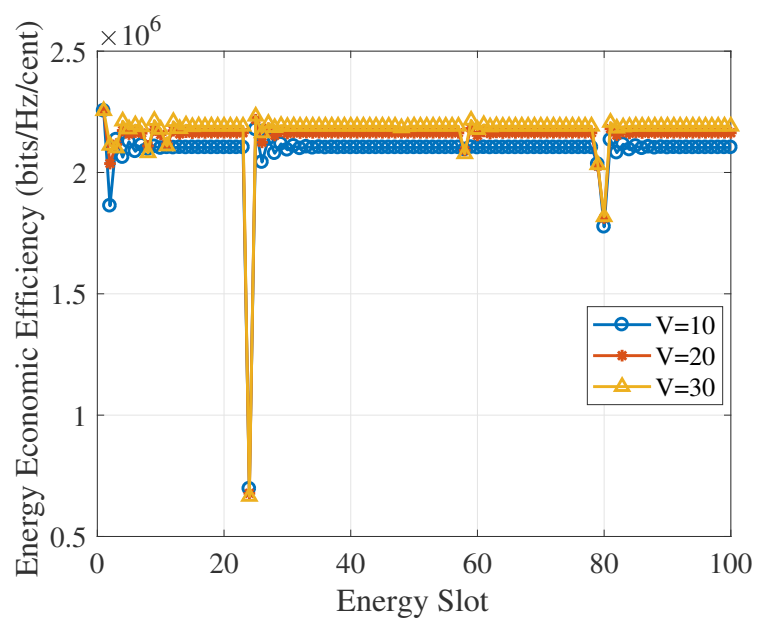

Fig. 25. A snapshot of energy economic efficiency.

Fig. 27 shows the convergence performance of Algorithm 2 under different simulation scenarios that $M=10,20,60,100$. Simulation results demonstrate the proposed algorithm only requires $3 \sim 4$ iterations for convergence, which is feasible for practical implementation.

\section{CONCLUSION}

In this paper, we studied the implementation of large-scale multi-antenna systems for smart grid. First, we developed a new performance benchmark named energy economic efficiency for measuring the time-average throughput per unit energy cost. Then, we developed an online 


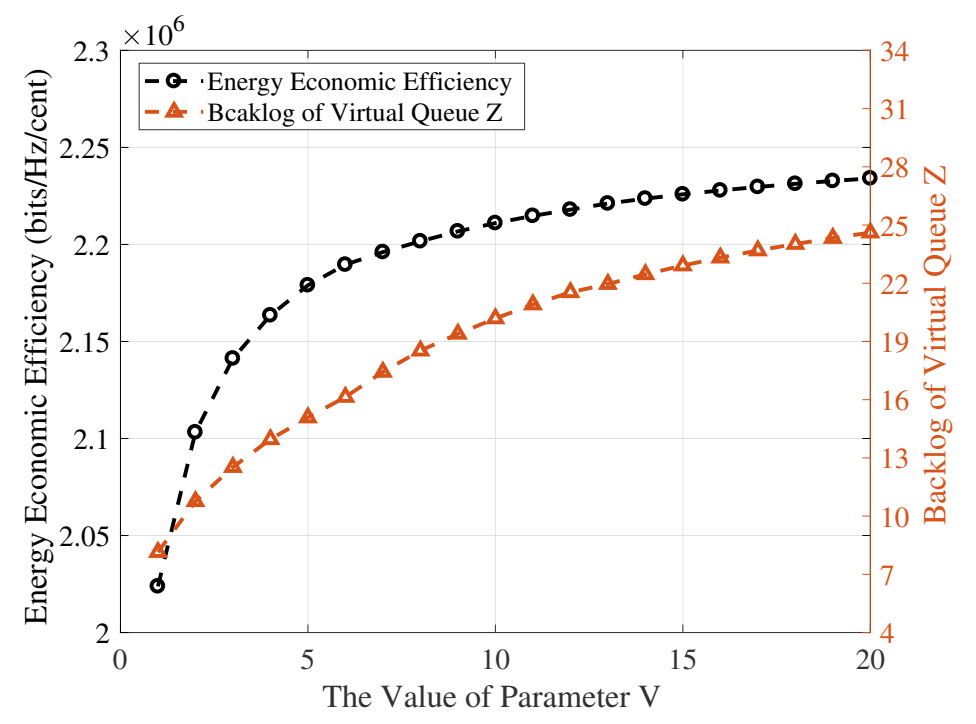

Fig. 26. Economic energy efficiency performance and backlog of queue $Z$ versus the parameter $V$.

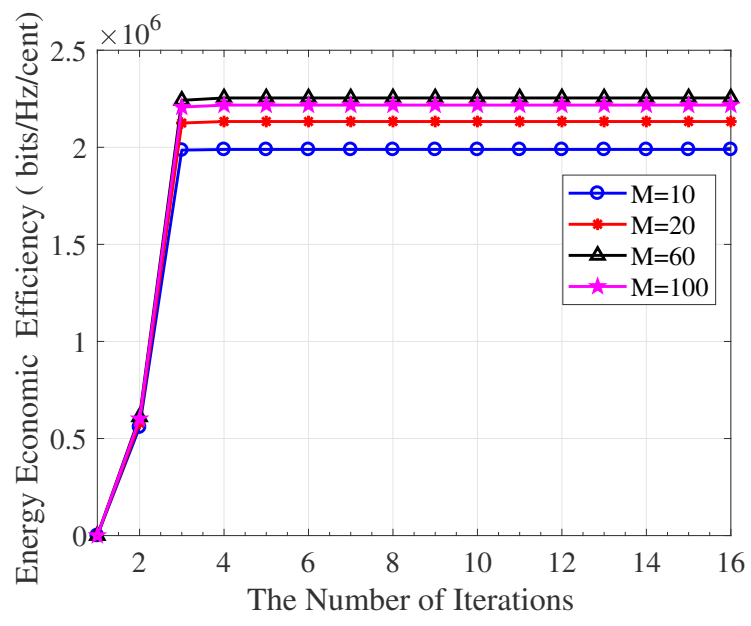

Fig. 27. The number of iterations $n$ of fractional optimization

joint antenna selection and power control algorithm to maximize the long-term energy economic efficiency performance by combining nonlinear fractional programming, Lyapunov optimization, and bisection method. We proved that the proposed algorithm can achieve bounded performance deviation from the optimum performance without requiring the prior knowledge of future energy arrival and electricity price. Simulation results show that the proposed algorithm outperforms the baseline 1 algorithm and baseline 2 algorithm by $73 \%$ and $552 \%$, respectively, when the battery 
capacity is sufficient. It is also observed that there exists a $[O(1 / V), O(V)]$ tradeoff between queue backlog and energy economic efficiency, and the proposed algorithm can dynamically adapt grid power consumption with time-varying electricity price. In the future work, we plan to study how to integrate learning capabilities with existing framework to further improve the performance.

\section{APPENDIX A}

\section{PROOF OF THEOREM 1}

According to (4), we have

$$
\begin{aligned}
I_{\text {sel }} & =\left.K \log _{2}\left|1+\frac{P_{T x}}{\sigma^{2} K^{2}} \sum_{j=1}^{M} \sum_{k=1}^{K}\right| h_{k, j}\right|^{2} \mid \\
& =K \log _{2}\left|\left[1+\frac{P_{T x}}{\sigma^{2} K}\left(1+\ln \frac{N}{M}\right)\right] r\right| \\
& =K\left[\log _{2}\left|1+\frac{P_{T x}}{\sigma^{2} K}\left(1+\ln \frac{N}{M}\right)\right|+\log _{2}|r|\right] \\
& =K\left[\log _{2}\left|1+\frac{P_{T x}}{\sigma^{2} K}\left(1+\ln \frac{N}{M}\right)\right|+\log _{2}(1+|r|-1)\right] \\
& =K\left[\log _{2}\left|1+\frac{P_{T x}}{\sigma^{2} K}\left(1+\ln \frac{N}{M}\right)\right|+(|r|-1) \log _{2} e\right],
\end{aligned}
$$

where $|\cdot|$ denotes the absolute value, and $r$ is given by

$$
r=1+\frac{\frac{P_{T x}}{\sigma^{2} K^{2}} \sum_{j=1}^{M} \sum_{k=1}^{K}\left|h_{k, j}\right|^{2}-\frac{P_{T x} M}{\sigma^{2} K}\left(1+\ln \frac{N}{M}\right)}{1+\left(1+\ln \frac{N}{M}\right) \frac{P_{T x} M}{\sigma^{2} K}} .
$$

According to [11], we have

$$
\sum_{j=1}^{M} \sum_{k=1}^{K}\left|h_{k, j}\right|^{2} \sim \mathcal{N}\left(K M\left(1+\ln \frac{N}{M}\right), K M\left(2-\frac{M}{N}\right)\right) .
$$

Then, combining (52) with (51), we can obtain

$$
r \sim \mathcal{N}\left(1, \frac{P_{T x}^{2} M\left(2-\frac{M}{N}\right)}{K^{3} \sigma^{4}\left[1+\left(1+\ln \frac{N}{M}\right) \frac{P_{T x} M}{K \sigma^{2}}\right]^{2}}\right) .
$$

Given a random variable $r$ with normal distribution, its absolute value $|r|$ follows a folded normal distribution [11]. Thus, the distribution of $I_{\text {sel }}$ is given by

$$
I_{s e l} \sim \mathcal{F N}\left(K \log _{2}\left[1+\left(1+\ln \frac{N}{M}\right) \frac{P_{T x} M}{K \sigma^{2}}\right], \frac{\left(\log _{2} e P_{T x}\right)^{2} M\left(2-\frac{M}{N}\right)}{\left.K \sigma^{4}\left[1+\left(1+\ln \frac{N}{M}\right) \frac{P_{T x} M}{K \sigma^{2}}\right)\right]^{2}}\right) .
$$




\section{APPENDIX B}

\section{Proof OF THE THEOREM 6}

Lemma 1. For arbitrary energy arrival rates, there exists a randomized control policy which achieves the following steady state values

$$
\begin{aligned}
\mathbb{E}\left\{P_{T X}\left(t_{e}\right)\right\} & \leq P_{T X, \text { mean }}-\epsilon, \\
\mathbb{E}\left\{C_{\text {total }}\left(\mathcal{S}_{t_{e}}^{*}, \mathcal{P}_{t_{e}}^{*}\right)-C_{\text {total }}\left(\mathcal{S}_{t_{e}}, \mathcal{P}_{t_{e}}\right)\right\} & \leq \delta \\
\mathbb{E}\left\{U_{\text {sel }}\left(\mathcal{S}_{t_{e}}, \mathcal{P}_{t_{e}}\right)\right\} & \geq \mathbb{E}\left\{C_{\text {total }}\left(\mathcal{S}_{t_{e}}, \mathcal{P}_{t_{e}}\right)\right\}\left(q^{*}-\delta\right) .
\end{aligned}
$$

Proof: A similar proof can be found in [45], [53].

Remark 5: It is noted that Lemma 1 still holds as long as the system state can take at most a finite (but arbitrarily large) number of values. Since the system state in our work depends on battery state, energy arrival, renewable energy price, and grid power. With discretization and quantification, we can guarantee that these values as well as the corresponding system state space are also finite.

Substituting (55) into (24) and taking the limit as $\delta \rightarrow 0$, we can get

$$
\begin{aligned}
& \Delta\left(Z\left(t_{e}\right)\right)-V \mathbb{E}\left\{U_{\text {sel }}\left(\mathcal{S}_{t_{e}}, \mathcal{P}_{t_{e}}\right)-q\left(t_{e}\right) C_{\text {total }}\left(\mathcal{S}_{t_{e}}, \mathcal{P}_{t_{e}}\right)\right\} \\
\leq & C-V q^{*} \mathbb{E}\left\{C_{\text {total }}\left(\mathcal{S}_{t_{e}}, \mathcal{P}_{t_{e}}\right)\right\}+V q\left(t_{e}\right) \mathbb{E}\left\{C_{\text {total }}\left(\mathcal{S}_{t_{e}}, \mathcal{P}_{t_{e}}\right)\right\}-\epsilon\left(Z\left(t_{e}\right)\right) .
\end{aligned}
$$

When $Z\left(t_{e}\right) \geq 0$, (56) can be further simplified as

$$
\begin{gathered}
\Delta\left(Z\left(t_{e}\right)\right)-V \mathbb{E}\left\{U_{\text {sel }}\left(\mathcal{S}_{t_{e}}, \mathcal{P}_{t_{e}}\right)-q\left(t_{e}\right) C_{\text {total }}\left(\mathcal{S}_{t_{e}}, \mathcal{P}_{t_{e}}\right)\right\} \\
\leq C-V q^{*} \mathbb{E}\left\{C_{\text {total }}\left(\mathcal{S}_{t_{e}}, \mathcal{P}_{t_{e}}\right)\right\}+V q\left(t_{e}\right) \mathbb{E}\left\{C_{\text {total }}\left(\mathcal{S}_{t_{e}}, \mathcal{P}_{t_{e}}\right)\right\}
\end{gathered}
$$

Applying the law of telescoping sums over $t_{e}=\left\{1,2, \cdots, T_{e} T_{\omega}\right\}$ and the law of iterated expectations, we have

$$
\begin{aligned}
& \mathbb{E}\left\{L\left(Z\left(T_{e} T_{\omega}\right)\right)\right\}-\mathbb{E}\{L(Z(1))\}-V \sum_{t_{e}=1}^{T_{e} T_{\omega}} \mathbb{E}\left\{U_{\text {sel }}\left(\mathcal{S}_{t_{e}}, \mathcal{P}_{t_{e}}\right)-q\left(t_{e}\right) C_{\text {total }}\left(\mathcal{S}_{t_{e}}, \mathcal{P}_{t_{e}}\right)\right\} \\
\leq & T_{e} T_{\omega}\left[C-V q^{*} \mathbb{E}\left\{C_{\text {total }}\left(\mathcal{S}_{t_{e}}, \mathcal{P}_{t_{e}}\right)\right\}\right]+V \mathbb{E}\left\{C_{\text {total }}\left(\mathcal{S}_{t_{e}}, \mathcal{P}_{t_{e}}\right)\right\} \sum_{t_{e}=1}^{T_{e} T_{\omega}} \mathbb{E}\left\{q\left(t_{e}\right)\right\}
\end{aligned}
$$


Dividing both sides of (58) by $V T_{e} T_{\omega}$, we can obtain

$$
\begin{aligned}
& \frac{1}{T_{e} T_{\omega}} \sum_{t_{e}=1}^{T_{e} T_{\omega}} \mathbb{E}\left\{q\left(t_{e}\right) C_{\text {total }}\left(\mathcal{S}_{t_{e}}, \mathcal{P}_{t_{e}}\right)-U_{\text {sel }}\left(\mathcal{S}_{t_{e}}, \mathcal{P}_{t_{e}}\right)\right\} \\
\leq & \frac{C}{V}-q^{*} \mathbb{E}\left\{C_{\text {total }}\left(\mathcal{S}_{t_{e}}, \mathcal{P}_{t_{e}}\right)\right\}+\mathbb{E}\left\{C_{\text {total }}\left(\mathcal{S}_{t_{e}}, \mathcal{P}_{t_{e}}\right)\right\} \frac{1}{T_{e} T_{\omega}} \sum_{t_{e}=1}^{T_{e} T_{\omega}} \mathbb{E}\left\{q\left(t_{e}\right)\right\}+\frac{\mathbb{E}\{L(Z(1))\}}{V T_{e} T_{\omega}} .
\end{aligned}
$$

Taking the limit $T_{\omega} \rightarrow \infty$, we can derive

$$
\begin{aligned}
& \lim _{T_{\omega} \rightarrow \infty} \frac{1}{T_{e} T_{\omega}} \sum_{t_{e}=1}^{T_{e} T_{\omega}} \mathbb{E}\left\{q\left(t_{e}\right) C_{\text {total }}\left(\mathcal{S}_{t_{e}}, \mathcal{P}_{t_{e}}\right)-U_{\text {sel }}\left(\mathcal{S}_{t_{e}}, \mathcal{P}_{t_{e}}\right)\right\} \\
= & \lim _{T_{\omega} \rightarrow \infty}\left[\frac{1}{T_{e} T_{\omega}} \sum_{t_{e}=1}^{T_{e} T_{\omega}} \mathbb{E}\left\{q\left(t_{e}\right) C_{\text {total }}\left(\mathcal{S}_{t_{e}}, \mathcal{P}_{t_{e}}\right)\right\}-\frac{1}{T_{e} T_{\omega}} \sum_{t_{e}=1}^{T_{e} T_{\omega}} \mathbb{E}\left\{U_{\text {sel }}\left(\mathcal{S}_{t_{e}}, \mathcal{P}_{t_{e}}\right)\right\}\right] \\
= & \overline{U_{\text {sel }}}\left(\mathcal{S}_{\text {te }}, \mathcal{P}_{t_{e}}\right)-\overline{U_{\text {sel }}}\left(\mathcal{S}_{\text {te }}, \mathcal{P}_{t_{e}}\right)=0 .
\end{aligned}
$$

Adding (60) into (59), we can obtain

$$
\frac{C}{V}-q^{*} \mathbb{E}\left\{C_{\text {total }}\left(\mathcal{S}_{t_{e}}, \mathcal{P}_{t_{e}}\right)\right\}+U_{E^{3}} \mathbb{E}\left\{C_{\text {total }}\left(\mathcal{S}_{t_{e}}, \mathcal{P}_{t_{e}}\right)\right\} \geq 0
$$

Rearranging (61), we have

$$
U_{E^{3}} \geq q^{*}-\frac{C}{V \mathbb{E}\left\{C_{\text {total }}\left(\mathcal{S}_{t_{e}}, \mathcal{P}_{t_{e}}\right)\right\}} \geq q^{*}-\frac{C}{V C_{\text {total,min }}},
$$

where $C_{\text {total,min }}$ is the minimum value of $C_{\text {total }}\left(\mathcal{S}_{t_{e}}, \mathcal{P}_{t_{e}}\right)$ over the entire period.

Similarly, applying the law of iterated expectations and the law of telescoping sums over $t_{e} \in\left\{1,2, \ldots, T_{e} T_{\omega}\right\}$ for (56), we can get

$$
\begin{gathered}
\mathbb{E}\left\{L\left(Z\left(T_{e} T_{\omega}\right)\right)\right\}-\mathbb{E}\{L(Z(1))\}-V \sum_{t_{e}=1}^{T_{e} T_{\omega}} \mathbb{E}\left\{U_{\text {sel }}\left(\mathcal{S}_{t_{e}}, \mathcal{P}_{t_{e}}\right)-q\left(t_{e}\right) C_{\text {total }}\left(\mathcal{S}_{t_{e}}, \mathcal{P}_{t_{e}}\right)\right\} \\
\leq T_{e} T_{\omega}\left[C-V q^{*} \mathbb{E}\left\{C_{\text {total }}\left(\mathcal{S}_{t_{e}}, \mathcal{P}_{t_{e}}\right)\right\}\right]+V \mathbb{E}\left\{C_{\text {total }}\left(\mathcal{S}_{t_{e}}, \mathcal{P}_{t_{e}}\right)\right\} \sum_{t_{e}=1}^{T_{e} T_{\omega}} \mathbb{E}\left\{q\left(t_{e}\right)\right\}-\epsilon \sum_{t_{e}=1}^{T_{e} T_{\omega}} Z\left(t_{e}\right) .
\end{gathered}
$$

Dividing the both sides of (63) by $\epsilon T_{e} T_{\omega}$ and taking the limit as $T_{\omega} \rightarrow \infty$, we can obtain

$$
\begin{aligned}
\bar{Z} & =\lim _{T_{\omega} \rightarrow \infty} \frac{1}{T_{e} T_{\omega}} \sum_{t_{e}=1}^{T_{e} T_{\omega}} Z\left(t_{e}\right) \\
& \leq \frac{C-V q^{*} \mathbb{E}\left\{C_{\text {total }}\left(\mathcal{S}_{t_{e}}, \mathcal{P}_{t_{e}}\right)\right\}}{\epsilon}+\frac{V}{\epsilon} \lim _{T_{\omega} \rightarrow \infty} \frac{1}{T_{e} T_{\omega}} \sum_{t_{e}=1}^{T_{e} T_{\omega}} \mathbb{E}\left\{q\left(t_{e}\right) C_{\text {total }}\left(\mathcal{S}_{t_{e}}, \mathcal{P}_{t_{e}}\right)\right\} \\
& \leq \frac{C+V\left(U_{\text {sel }, \text { max }}-q^{*} C_{\text {total,min }}\right)}{\epsilon}
\end{aligned}
$$

This completes the proof. 


\section{REFERENCES}

[1] T. L. Marzetta, "Noncooperative cellular wireless with unlimited number of base station antennas," IEEE Trans. Wireless Commun., vol. 9, no. 11, pp. 3590-3600, Nov. 2010.

[2] X. Yu, J. Zhang, M. Haenggi, and K. B. Letaief, "Coverage analysis for millimeter wave networks: The impact of directional antenna arrays," IEEE J. Sel. Areas Commun., vol. 35, no. 7, pp. 1498-1512, Apr. 2017.

[3] J. Ma, S. Zhang, H. Li, N. Zhao, and V. C. M. Leung, "Interference-alignment and soft-space-reuse based cooperative transmission for multi-cell massive MIMO networks," IEEE Trans. Wireless Commun., vol. 17, no. 3, pp. 1907-1922, Mar. 2018.

[4] H. Q. Ngo, E. G. Larsson, and T. L. Marzetta, "Energy and spectral efficiency of very large multiuser MIMO systems," IEEE Trans. Commun., vol. 61, no. 4, pp. 1-14, Apr. 2013.

[5] X. Gao, O. Edfors, F. Tufvesson, and E. G. Larsson, "Massive MIMO in real propagation environments: Do all antennas contribute equally," IEEE Trans. Commun., vol. 63, no. 11, pp. 3917-3928, Nov. 2015.

[6] E. G. Larsson, O. Edfors, F. Tufvesson, and T. L. Marzetta, "Massive MIMO for next generation wireless systems," IEEE Commun. Mag., vol. 52, no. 2, pp. 186-195, Feb. 2014.

[7] A. F. Molisch and M. Z. Win, "MIMO systems with antenna selection," IEEE Microw. Mag., vol. 5, no. 1, pp. 46-56, Mar. 2004.

[8] J. Ma, S. Zhang, H. Li, F. Gao, and S. Jin, "Sparse Bayesian learning for the time-varying massive MIMO channels: Acquisition and tracking," IEEE Trans. Commun., vol. 67, no. 3, pp. 1925-1938, Mar. 2019.

[9] H. L. et. al, "Learning-based context-aware resource allocation for edge computing-empowered industrial iot," IEEE Internet Things J., pp. 1-1, 2019.

[10] D. W. K. Ng, E. S. Lo, and R. Schober, "Energy-efficient resource allocation in OFDMA systems with hybrid energy harvesting base station," IEEE Trans. Wireless Commun., vol. 12, no. 7, pp. 3412-3427, Jul. 2013.

[11] H. Li, L. Song, and M. Debbah, "Energy efficiency of large-scale multiple antenna systems with transmit antenna selection," IEEE Trans. Commun., vol. 62, no. 2, pp. 638-647, Feb. 2014.

[12] P. V. Amadori and C. Masouros, "Interference-driven antenna selection for massive multiuser MIMO," IEEE Trans. Veh. Technol., vol. 65, no. 8, pp. 5944-5958, Aug. 2016.

[13] C. He, G. Y. Li, F. Zheng, and X. You, "Energy-efficient resource allocation in OFDM systems with distributed antennas," IEEE Trans. Veh. Technol., vol. 63, no. 3, pp. 1223-1231, Mar. 2014.

[14] D. Gonzalez, D. Costa, and J. Filho, "Distributed TAS/MRC and TAS/SC schemes for fixed-gain AF systems with multiantenna relay: Outage performance,” IEEE Trans. Wireless Commun., vol. 15, no. 6, pp. 1536-1276, Jun. 2016.

[15] J. Yang and S. Ulukus, "Optimal packet scheduling in an energy harvesting communication system," IEEE Trans. Commun., vol. 60, no. 1, pp. 220-230, Jan. 2012.

[16] M. A. Antepli, E. Uysal-Biyikoglu, and E. Erkal, "Optimal packet scheduling on an energy harvesting broadcast link," IEEE J. Sel. Areas Commun., vol. 29, no. 8, pp. 1712-1731, Sep. 2011.

[17] C. Xu, M. Zheng, W. Liang, H. Yu, and Y. C. Liang, "End-to-end throughput maximization for underlay multi-hop cognitive radio networks with RF energy harvesting,” IEEE Trans. Wireless Commun., vol. 16, no. 6, pp. 3561-3572, Mar. 2017.

[18] C. Huang, R. Zhang, and S. Cui, "Throughput maximization for the Gaussian relay channel with energy harvesting constraints," IEEE J. Sel. Areas Commun., vol. 31, no. 8, pp. 1469-1479, Aug. 2013.

[19] J. Yang, O. Ozel, and S. Ulukus, "Broadcasting with an energy harvesting rechargeable transmitter," IEEE Trans. Wireless Commun., vol. 11, no. 2, pp. 571-583, Feb. 2012. 
[20] F. Benkhelifa, A. S. Salem, and M. S. Alouini, "Sum-rate enhancement in multiuser MIMO decode-and-foward relay broadcasting channel with energy harvesting relays," IEEE J. Sel. Areas Commun., vol. 34, no. 12, pp. 3675-3684, Dec. 2016.

[21] R. G. Stephen, C. R. Murthy, and M. Coupechoux, "A Markov decision theoretic approach to pilot allocation and receive antenna selection," IEEE Trans. Wireless Commun., vol. 12, no. 8, pp. 3813-3823, Aug. 2013.

[22] J. Gong, S. Zhou, Z. Zhou, and Z. Niu, "Policy optimization for content push via energy harvesting small cells in heterogeneous networks," IEEE Trans. Wireless Commun., vol. 16, no. 2, pp. 719-729, Feb. 2017.

[23] J. Gong, S. Zhou, and Z. Zhou, "Networked MIMO with fractional joint transmission in energy harvesting systems," IEEE Trans. Commun., vol. 64, no. 8, pp. 3323-3336, Aug. 2016.

[24] V. Sharma, U. Mukherji, V. Joseph, and S. Gupta, "Optimal energy management policies for energy harvesting sensor nodes," IEEE Trans. Wireless Commun., vol. 9, no. 4, pp. 1326-1336, Apr. 2010.

[25] D. P. Bertsekas, Dynamic Programming and Optimal Control. Cambridge, MA, USA: Athena Scientific, 2006.

[26] Z. Jiang, S. Zhou, and Z. Niu, "A block coordinated update method for beam-based massive MIMO downlink scheduling based on statistical CSI," in Proc. Asia-Pacific Conf. Commun. (APCC), Perth, WA, Australia, Dec. 2017.

[27] Z. Jiang, C. Sheng, Z. Sheng, and Z. Niu, "Joint user scheduling and beam selection optimization for beam-based massive MIMO downlinks," IEEE Trans. Wireless Commun., vol. 17, no. 99, pp. 2190-2204, Apr. 2017.

[28] Y. Mao, J. Zhang, and K. B. Letaief., "A Lyapunov optimization approach for green cellular networks with hybrid energy supplies," IEEE J. Sel. Areas Commun., vol. 33, no. 12, pp. 2463-2477, Dec. 2015.

[29] O. T. Demir and T. E. Tuncer, "Antenna selection and hybrid beamforming for simultaneous wireless information and power transfer in multi-group multicasting systems," IEEE Trans. Wireless Commun., vol. 15, no. 10, pp. 6948-6962, Oct. 2016.

[30] G. Amarasuriya, E. G. Larsson, and H. V. Poor, "Wireless information and power transfer in multiway massive MIMO relay networks," IEEE Trans. Wireless Commun., vol. 15, no. 6, pp. 3837-3855, Jun. 2016.

[31] L. Chen, F. R. Yu, H. Ji, B. Rong, X. Li, and V. C. M. Leung, "Green full-duplex self-backhaul and energy harvesting small cell networks with massive MIMO,” IEEE J. Sel. Areas Commun., vol. 34, no. 12, pp. 3709-3724, Dec. 2016.

[32] P. He, Z. Lian, and B. Venkatesh, "Novel water-filling for maximum throughput of power grid, MIMO and energy harvesting coexisting system with mixed constraints," IEEE Trans. Commun., vol. 65, no. 2, pp. 827-838, Feb. 2017.

[33] Z. Zhou, S. Zhou, J. Gong, and Z. Niu, "Energy-efficient antenna selection and power allocation for large-scale multiple antenna systems with hybrid energy supply," in Proc. IEEE Global Commun. Conf. (GLOBECOM), Austin, TX, USA, Dec. 2014, pp. 1-6.

[34] J. Men, J. Ge, and C. Zhang, "A joint relay-and-antenna selection scheme in energy-harvesting MIMO relay networks," IEEE Signal Process. Lett., vol. 23, no. 4, pp. 532-536, Apr. 2016.

[35] Y. Cui, V. K. N. Lau, and F. Zhang, "Grid power-delay tradeoff for energy harvesting wireless communication systems with finite renewable energy storage," IEEE J. Sel. Areas Commun., vol. 33, no. 8, pp. 1651-1666, Aug. 2015.

[36] C. Wu, Q. Shi, C. He, and Y. Chen, "Energy utilization efficient frame structure for energy harvesting cognitive radio networks," IEEE Wireless Commun. Lett., vol. 5, no. 5, pp. 488-491, Oct. 2016.

[37] B. Xu, Y. Chen, J. R. Carrión, and T. Zhang, "Resource allocation in energy-cooperation enabled two-tier NOMA hetnets toward green 5G," IEEE J. Sel. Areas Commun., vol. 35, no. 12, pp. 2758-2770, Dec. 2017.

[38] M. Jung, T. Kim, K. Min, Y. Kim, J. Lee, and S. Choi, "Asymptotic distribution of system capacity in multiuser MIMO systems with large number of antennas," in Proc. IEEE 77th Veh. Technol. Conf., Dresden, Germany, Jun. 2013, pp. 1-5.

[39] B. M. Hochwald, T. L. Marzetta, and V. Tarokh, "Multiple-antenna channel hardening and its implications for rate feedback and scheduling," IEEE Trans. Inf. Theory, vol. 50, no. 9, pp. 1893-1909, Sep. 2004. 
[40] Y. Gao, H. Vinck, and T. Kaiser, "Massive MIMO antenna selection: Switching architectures, capacity bounds, and optimal antenna selection algorithms," IEEE Trans. Signal Process., vol. 66, no. 5, pp. 1346-1360, Mar. 2018.

[41] K. Tourki, F. A. Khan, K. A. Qaraqe, H. Yang, and M. Alouini, "Exact performance analysis of MIMO cognitive radio systems using transmit antenna selection," IEEE J. Sel. Areas Commun., vol. 32, no. 3, pp. 425-438, Mar. 2014.

[42] Y. Hei, Y. Liu, W. Li, J. Liu, and X. Li, "Energy efficiency optimisation of large-scale multiple-input-multiple-output system with transmit antenna selection,” IET Commun., vol. 11, no. 8, pp. 1224-1229, Jun. 2017.

[43] G. Auer, "D2.3: Energy efficiency analysis of the reference systems, areas of improvements and target breakdown," Tech. Rep. INFO-ICT-247733 EARTH, Ver. 2.0 2012. [Online]. Available: http://www.ict-earth.eu/.

[44] H. Zhang, B. Wang, C. Jiang, K. Long, A. Nallanathan, V. C. M. Leung, and H. V. Poor, "Energy efficient dynamic resource optimization in NOMA system," IEEE Trans. Wireless Commun., vol. 17, no. 9, pp. 5671-5683, Sep. 2018.

[45] Y. Li, M. Sheng, Y. Shi, X. Ma, and W. Jiao, "Energy efficiency and delay tradeoff for time-varying and interference-free wireless networks,” IEEE Trans. Wireless Commun., vol. 13, no. 11, pp. 5921-5931, Nov. 2014.

[46] K. Yang, S. Martin, D. Quadri, J. Wu, and G. Feng, "Energy-efficient downlink resource allocation in heterogeneous OFDMA networks," IEEE Trans. Veh. Technol., vol. 66, no. 6, pp. 5086-5098, Jun. 2017.

[47] S. Boyd and L. Vandenberghe, Convex Optimization. Cambridge, UK: Cambridge University Press, 2004, pp. $144-191$.

[48] B. S. Krongold, K. Ramchandran, and D. L. Jones, "Computationally efficient optimal power allocation algorithms for multicarrier communication systems," IEEE Trans. Commun., vol. 48, no. 1, pp. 23-27, Jan. 2000.

[49] P. Tsiaflakis, I. Necoara, J. A. K. Suykens, and M. Moonen, "Improved dual decomposition based optimization for DSL dynamic spectrum management," IEEE Trans. Signal Process., vol. 58, no. 4, pp. 2230-2245, Apr. 2010.

[50] S. Boyd, L. Xiao, and A. Mutapcic, "Subgradient methods," Notes for EE392o Stanford University Autumn 2003, pp. 1-21, Oct. 2003.

[51] C. Jiang and L. J. Cimini, "Antenna selection for energy-efficient MIMO transmission," IEEE Wireless Commun. Lett., vol. 1, no. 6, pp. 577-580, Dec. 2012.

[52] Z. Zhou, Y. Guo, Y. He, X. Zhao, and W. M. Bazzi, "Access control and resource allocation for M2M communications in industrial automation,” IEEE Trans. Ind. Informat., vol. 15, no. 5, pp. 3093-3103, May 2019.

[53] M. J. Neely, Stochastic Network Optimization with Application to Communication and Queueing Systems. USA: Morgan \& Claypool, 2010, pp. 1-211. 
2020-07-28

Power control optimization for large-scale multi-antenna systems

Zhou, Zhenyu

IEEE

Yu H, Zhou Z, Mumtaz S, et al., (2020) Power control optimization for large-scale multi-antenna systems. IEEE Transactions on Wireless Communications, Volume 19, Issue 11, November 2020, pp.7339 - 7352

https://doi.org/10.1109/TWC.2020.3010701

Downloaded from Cranfield Library Services E-Repository 